\title{
Partitioned Time-Domain Substructure Coupling Methodology for Efficient Hypersonic Vehicle Simulation
}

\author{
Nathan J. Falkiewicz* and Carlos E. S. Cesnik \\ University of Michigan, Ann Arbor, Michigan 48109-2140
}

DOI: $\underline{10.2514 / 1 . J 051614}$

\begin{abstract}
A partitioned solution framework is developed for time-domain aerothermoelastic simulation of hypersonic vehicles. The motivation for development of such an approach stems from the fact that hypersonic vehicles consist of multiple substructures, each containing different dominant physics. Different model forms are therefore required to capture the main physics of each substructure, and direct coupling of the various substructures of the vehicle is not straightforward. The methodology of this paper is based on a partitioned time-marching formulation, in which individual components of the vehicle are modeled separately, and forces/motion at the interface are exchanged between the systems within each time step. This approach is advantageous in that it does not require direct coupling of the substructures, therefore allowing for the models to be of dissimilar form. The methodology is applied to a representative configuration consisting of an all-movable hypersonic vehicle lifting surface model containing aerothermoelastic effects attached to a single-degree-of-freedom oscillator representing the fuselage. Results from validation cases are first presented. The partitioned solution methodology is then used to investigate the impact of lifting surface-fuselage inertial coupling on overall vehicle dynamics.
\end{abstract}

\section{Nomenclature}

$B$

$c$

$c_{p}$

$d$

E

F

$f$

$f_{i}$

$g$

$H_{i}$

$H(t)$

$h$

$h_{i}$

$I_{i}$

K

$K_{G}=$ physical geometric stiffness matrix

$K^{*}$

$k$

$k^{*}$

$\mathcal{L}$

$M$

$M_{y}^{W / B}$

$m$
$Q_{i}$

$q_{i}$

$R$

body-fixed reference frame

generalized damping matrix

$=$ specific heat

$=$ physical loads

$=$ generalized loads

$=$ gravitational acceleration scheme

$=$ Heaviside step function

$=$ altitude

$=$ generalized stiffness matrix

$=$ Lagrangian fuselage through attachment point

$=$ generalized mass matrix

$=$ residual
$=$ control surface elastic modal coordinates

$=$ modulus of elasticity, Earth-fixed reference frame

$=$ fuselage ordinary natural frequencies

$=$ coefficient matrices in control surface integration

$=$ thickness of $i$ th layer of thermal protection system

$=$ coefficient matrices in fuselage integration scheme

$=$ physical conventional stiffness matrix, stiffness of

$=$ physical modified structural stiffness matrix

$=$ generalized modified stiffness matrix

$=$ physical mass matrix, Mach number, fuselage mass

$=$ pitching moment exerted by control surface on

$=i$ th generalized force acting on fuselage

$=$ arbitrary generalized coordinate

Presented as Paper 2011-6378 at the AIAA Atmospheric Flight Mechanics Conference, Portland, OR, 8-11 August 2011; received 23 September 2011; revision received 23 November 2014; accepted for publication 26 May 2015; published online 10 September 2015. Copyright @ 2014 by N. J. Falkiewicz and C. E. S. Cesnik. Published by the American Institute of Aeronautics and Astronautics, Inc., with permission. Copies of this paper may be made for personal or internal use, on condition that the copier pay the $\$ 10.00$ per-copy fee to the Copyright Clearance Center, Inc., 222 Rosewood Drive, Danvers, MA 01923; include the code 1533-385X/15 and $\$ 10.00$ in correspondence with the CCC.

*Ph.D. Candidate, Department of Aerospace Engineering, 1320 Beal Avenue; currently Technical Staff, Lincoln Laboratory, Massachusetts Institute of Technology, Lexington, MA 02420. Member AIAA.

${ }^{\dagger}$ Professor of Aerospace Engineering, Department of Aerospace Engineering, 1320 Beal Avenue. Fellow AIAA.
$T$

$\mathcal{T}_{0}=$

$t=$

$U \quad=$

$V \quad=$

$w_{a} \quad=$

$x=$

$\alpha \quad=$

$\alpha_{\text {net }}=$

$\alpha_{T} \quad=$

$\beta=$

$\Delta t$

$\delta$

$\zeta_{\delta}$

$\zeta_{\delta}$

$\eta$

$\kappa$

$\mu_{i}$

$\nu$

$\rho \quad=$

$\tau \quad=$

$\Phi \quad=$

$\omega_{i} \quad=$

$\omega_{\delta} \quad=$

Subscripts

$\mathrm{AE}=$

$\mathrm{AE} \quad=$ aeroelastic

AT $=$ aerothermal

$B \quad=$ body fixed

cmd $=$ commanded value

$d=$ time instant at which $\alpha$ is decremented

$f \quad=$ fuselage

HT $=$ heat transfer

$i=$ time instant at which $\alpha$ is incremented

$\max =$ maximum application temperature

$r \quad=$

$T \quad=$ component due to thermal loads unrestrained degrees of freedom time duration for increment in angle of attack generalized load associated with fuselage rigid-body translational velocity 
$\zeta=$ generalized load associated with fuselage rigid-body rotational velocity

$\eta \quad=$ generalized load associated with fuselage elastic degrees of freedom

$\infty \quad=$ freestream

\section{Superscripts}

A $=$ component of structural loads due to aerodynamic pressure

$C=$ constraint motion

$E \quad=$ elastic displacement

$H=$ component of structural loads due to heating

(n) = time level

$W \quad=$ force component due to control surface (wing) motion

$W / B=$ net external force/moment imposed by control surface (wing) relative to fuselage (body)

$-1=$ matrix inverse

\section{Introduction}

D ESIGN and simulation of hypersonic vehicles (HSVs) require consideration of a variety of disciplines due to the highly coupled nature of their flight regime [1]. The highly integrated nature of hypersonic flight stems from various factors specific to these types of vehicles. Airbreathing hypersonic vehicles typically consist of a tightly integrated airframe along with a scramjet propulsion system. The forward fuselage of the vehicle represents the compression ramp, which produces the necessary flow conditions for the inlet of the propulsion system. This results in a pressure distribution that causes a nose-up pitching moment. The aft section of the vehicle consists of an external exhaust nozzle shaped to allow for expansion of the flow exiting the engine. Additionally, the location of the engine below the vehicle center of gravity results in a nose-up pitching moment due to thrust, which must be balanced [2]. Further complicating the coupling between the propulsion system and the airframe are the elastic deformations of the forebody and vehicle pitch response, which affect the inlet conditions to the engine [3]. Thus, to assess the overall vehicle performance, the effect of flexibility must be considered.

In addition to the preceding effects, aerodynamic heating due to flow stagnation, flow compression, and boundary-layer friction can also have a significant impact on HSVs [1, $\underline{4}]$. Hypersonic vehicles with airbreathing propulsion systems must fly at relatively low altitudes to maintain the dynamic pressure required for optimal engine performance [4]. One consequence of this requirement is that the high dynamic pressure and high Reynolds number lead to surface heating becoming a major design driver. The surface heating in turn leads to heat being conducted through the internal vehicle structure. The spatial variation of temperature throughout the structure leads to a change in stiffness distribution through two effects: temperaturedependent material properties and geometric stiffening effects due to internal thermal stresses. The effect of aerodynamic heating on the lifting surfaces is particularly important to consider because the lifting surfaces experience a large variation in temperature and the resulting change in stiffness and the deformation due to thermal loads can alter the vehicle flight dynamics.

Flight dynamic simulation of HSVs is further complicated by the large computational expense involved in capturing all of these disciplines and their interactions in a full-order sense. Although highfidelity modeling techniques exist for each of these disciplines, the use of such techniques is computationally infeasible in a vehicle design and simulation setting for such a highly coupled problem. Early in the design stage, many iterations of analyses may need to be carried out as the vehicle design matures, thus requiring quick analysis turnaround time. Additionally, the number of states and number of degrees of freedom (DOF) used in the analyses must be small enough to allow for efficient control simulation and design. As a result, alternative approaches must be considered for vehicle simulations. There are two methodologies that can be used in the generation of computationally efficient models. The first approach is to apply simplifying assumptions that enable the use of fundamental models (for example, the use of an analytical beam representation as opposed to a high-fidelity finite element model). These models can often be solved analytically, thus preventing the need to time march the solution. The second approach involves the use of reduced-order models (ROMs) that are derived from high-fidelity analysis tools. Use of high-fidelity tools alone is infeasible due to their high order and long run time. However, by using the output of these tools along with reduced-order modeling techniques, computationally tractable systems of governing equations with low numbers of states can be obtained. In this study, both fundamental models and reduced-order models are used to perform HSV flight dynamic simulations.

As a result of the complex coupling between multiple disciplines exhibited in hypersonic flight, as well as the need to employ reducedorder or analytical models, different model forms are required to capture the dominant physics of each of the various vehicle substructures. Aerodynamic heating is expected to have a strong impact on the response of the lifting surfaces, whereas its effect on the fuselage will likely not be as pronounced. The scramjet propulsion system consists of an inlet, isolator, combustor, and nozzle and contains a different set of physics than that associated with the fuselage and lifting surface structures. Therefore, direct or monolithic coupling of the vehicle substructures in the time-domain simulation is not straightforward. This paper focuses on the development of a partitioned solution methodology that allows for independent modeling of each of the vehicle substructures by coupling them through the exchange of interface information. By modeling the vehicle in this way, each substructure can be treated as a black box with respect to the others, thus facilitating coupling between the substructures.

The challenges associated with flight dynamics and control analysis of air-breathing HSVs have been reviewed in the literature $[5,6]$. To address these challenges, previous research into the flight dynamics of hypersonic vehicles has largely used analytical models of the various disciplines. The closed-form nature of these models allows for characterization of the vehicle dynamics early in the design cycle and permits evaluation of stability derivatives more readily than with numerical models. The first effort to develop a comprehensive analytical model was conducted by Chavez and Schmidt [3] . That work used Newtonian impact theory for the aerodynamic pressures, one-dimensional (1-D) aero-/thermoanalysis for the propulsion system, and a lumped-mass modal model for the structural dynamics. The methodology was applied to a two-dimensional (2-D) hypersonic vehicle geometry in which the control effectors consisted of aerodynamic pitch-control surfaces as well as the engine fuel flow and diffuser area ratio. The derived equations of motion (EOMs) were linearized, and analytical expressions were obtained for the stability and control derivatives.

A subsequent work [7] employed a Lagrangian approach to capture the elastic deformation, fluid flow, rotating machinery, and spherical Earth. The resulting equations of motion governing the rigid body and elastic degrees of freedom were derived and a preliminary study of the significance of selected terms in the equations was presented. A three-degree-of-freedom, point-mass dynamic model was also outlined and the equations were presented. For a single-stage-to-orbit configuration, the Coriolis force was found to reach values up to $6 \%$ of the vehicle weight. Another work [2] presented a nonlinear physics-based model of the longitudinal dynamics for an airbreathing HSV. Oblique shock and PrandtlMeyer expansion theory were used for the aerodynamics and the structure was modeled as two cantilever beams clamped at the center of mass of the fuselage. The vehicle analyzed in [2] included an elastic fuselage and elevator control surfaces that were modeled as rigid flat plates hinged at their midchord points. The equations of motion were derived and linearized to assess vehicle stability and coupling between the rigid-body and elastic dynamics. Results demonstrated that the linearized aircraft dynamics are unstable and exhibit nonminimum phase behavior in most cases. Coupling between the short-period mode and fuselage bending mode was also exhibited. 
In another approach [8], a closed-form, control-oriented model was obtain by replacing complex force and moment functions from a truth model with curve-fitted approximations. The resulting system was used to demonstrate an example control design based on approximate feedback linearization. The inclusion of additional flexible effects was found to render the original control design ineffective and an additional actuator was needed to enhance the control authority of the vehicle.

Although the preceding papers were successful in developing comprehensive models of hypersonic vehicles for flight dynamic analysis, they did not include the structural dynamics of the control surfaces as well as the resulting inertial coupling between those surfaces and the fuselage. The effect of such coupling can be important because it may result in a complex-conjugate pair of zeros in the elevator-to-pitch rate transfer function, thus altering the speed of response of the vehicle [9]. As such, the two main goals of the current work are as follows:

1) Develop a partitioned solution methodology such that vehicle substructures can be modeled independently and coupled in the time domain by exchanging interface information. This formulation allows for efficient modeling of lifting surface structural dynamics within a hypersonic vehicle framework to address the shortcomings discussed earlier. Demonstrate and validate the methodology using a configuration consisting of an all-movable hypersonic vehicle lifting surface model containing aerothermoelastic effects attached to a single-degree-of-freedom oscillator representing the fuselage.

2) Once validated, apply the partitioned solution methodology to the representative configuration described earlier to investigate the effect of lifting surface-fuselage inertial coupling on HSV dynamics.

\section{Simulation Framework}

The simulation framework of the current work is based on a partitioned approach in which the equations of motion for each substructure are integrated separately and information is exchanged between them at predetermined time intervals. In this work, the methodology is focused on the coupling between a hypersonic vehicle lifting surface and fuselage. The advantage of using separate models for the fuselage and lifting surfaces is that each model can be tailored specific to the physics of interest for that component. In the full vehicle simulation, the equations of motion for the fuselage are derived in analytical form by approximating it as a 1-D beam, whereas those for the lifting surfaces are based on a finite element discretization. The effect of aerodynamic heating on the lifting surfaces is expected to be strong in comparison with its effect on the fuselage. As such, aerodynamic heating is only included in the lifting surface model. Therefore, the fuselage model will be dissimilar in form with respect to the lifting surface model, and a straightforward monolithic coupling is not easily facilitated. The use of independent models for the fuselage and lifting surface with information being exchanged between the two at the interface is advantageous in that it does not require a direct coupling between the two components. Additionally, the use of separate models for the fuselage and lifting surfaces allows for different time-integration schemes and time steps to be employed for each based on the expected timescales of the system dynamics. To couple the structures, interface information must be exchanged between the fuselage and lifting surfaces at specified intervals. Information is passed to the lifting surface equations of motion in the form of fuselage accelerations and displacements for the degrees of freedom at the interface. Information is passed back to the fuselage equations of motion in the form of forces exerted by the lifting surface on the fuselage at the interface.

The aeroelastic framework for the flexible fuselage response has been developed by Frendreis and Cesnik [10]. A flowchart of this framework is given in Fig. 1. The structural model of the fuselage consists of a structural representation and appropriate boundary conditions (BCs). The structural representation is taken to be an analytical model based on an Euler-Bernoulli beam. The boundary conditions are chosen to reflect a structure in free flight, such as freefree boundary conditions for a beam. The unsteady aerodynamic model is composed of a steady shock-expansion component with a piston theory correction to account for unsteady effects. In addition to aerodynamic loads, the fuselage also experiences propulsive loads, which are determined with a scramjet model. Because the lifting surfaces are not directly included in the fuselage aeroelastic analysis, their contribution is expressed as a set of resultant forces and moments applied at their attachment points. These loads are determined within the lifting surface aerothermoelastic framework, which is described subsequently. The coupled rigid-body/structural equations of motion under loading from unsteady aerodynamics, propulsion, and the lifting surfaces provide the rigid-body and structural responses of the fuselage. These are then used to determine the prescribed root motion of the lifting surface, which is passed to the lifting surface aerothermoelastic model.

To accurately capture the contributions of the lifting surfaces to the overall vehicle dynamics, aerothermoelastic effects on the lifting surfaces must be included. The aerothermoelastic model of the lifting surface used in the present paper is based on a reduced-order modeling framework developed by Falkiewicz et al. [11-15]. A flowchart of the aerothermoelastic framework used to model the lifting surfaces is given in Fig. 2. The process begins with the calculation of the heat flux at the outer surface of the structure at initial time using the Eckert reference temperature method [16]. With the boundary conditions and initial conditions of the thermal problem known, the transient temperature distribution is marched forward in time. Solution of the heat transfer problem is carried out in modal space using modes from proper orthogonal decomposition (POD) to avoid the computational cost of running full-order finite element analysis. Bypassing of the full-order thermal solution via the reduced-order solution is indicated by the gray blocks. The structural boundary conditions for the lifting surface are determined by its

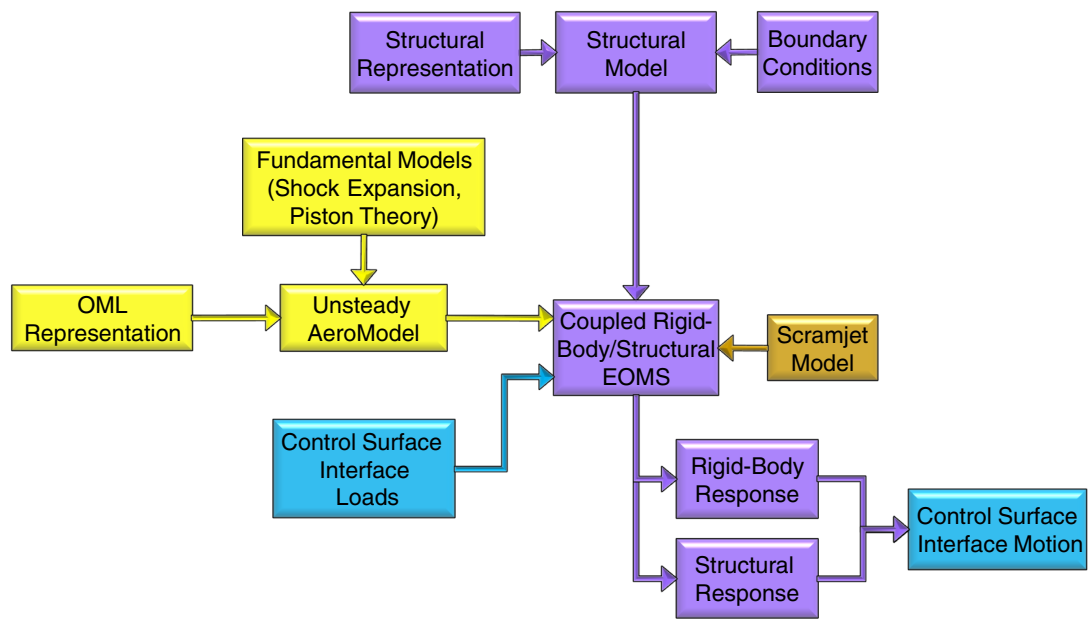

Fig. 1 Fuselage aeroelastic simulation framework. 


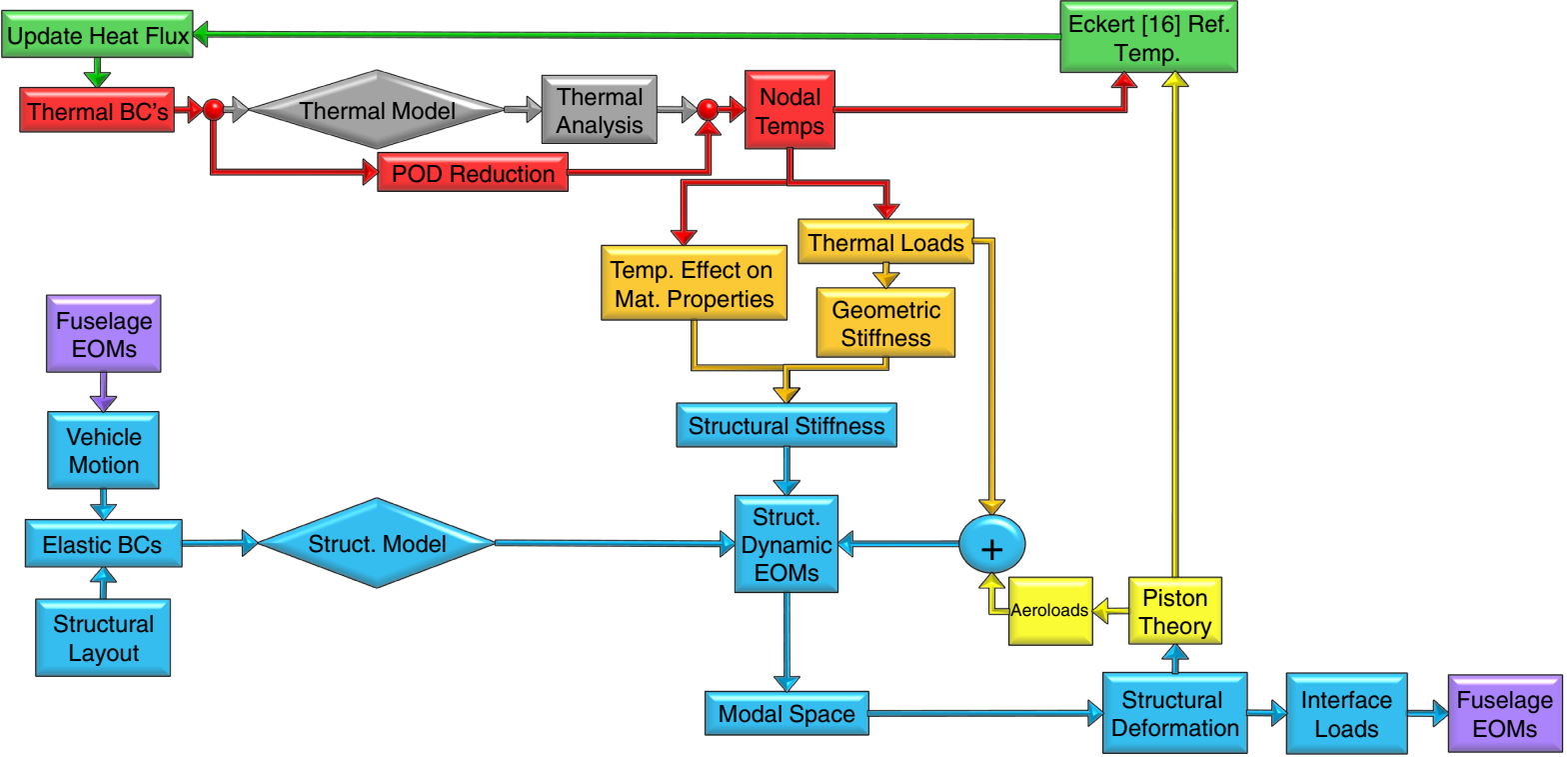

Fig. 2 Reduced-order aerothermoelastic modeling framework for lifting surface model.

layout as well as the fuselage motion at the interface, which comes from the solution of the fuselage equations of motion. This framework considers two coupling mechanisms between the thermal solution and the structural stiffness. The first involves the geometric stiffness effects due to thermal stresses that result from thermal expansion of the structure. The second is due to the temperature dependence of the Young's modulus resulting from the high temperatures experienced in hypersonic flight. In addition to thermal effects on the geometric stiffness, the change in temperature also results in thermal loads being applied to the structure.

With the stiffness and structural loads known, the structural dynamics system of equations in physical space is transformed to a suitable reduced modal basis. The reduced modal system is then solved for the modal coordinates to obtain the structural dynamic response. Once the response is known, it is used to compute the interface loads that the lifting surface exerts on the fuselage. These interface loads are passed to the fuselage equations of motion and represent force components at the degrees of freedom located at the lifting surface attachment point. The structural deformations of the lifting surface couple with the aerothermal problem due to the effect on aerodynamic flow properties, which change the heat flux. The deformations also result in a change in aerodynamic pressures, which modify the structural loads. With the deformed configuration known at the current time step, the unsteady aerodynamic flow properties are updated using third-order piston theory and the process is repeated at the next time step. After a predetermined number of aeroelastic iterations have been carried out, the heat flux boundary conditions are recalculated and the thermal solution is updated.
A time-marching procedure with updates to the thermal and structural boundary conditions at specified intervals is used for computing the aerothermoelastic response of the lifting surfaces. An outline of the time-stepping schedule is given in Fig. 3. The size of the aeroelastic time step, $\Delta t_{\mathrm{AE}}$, is smaller than the size of the aerothermal time step, $\Delta t_{\mathrm{AT}}$, because the aeroelastic timescale is faster than the thermal timescale. The procedure begins by calculating the aerodynamic flow properties over the undeformed structure at initial time, $t_{0}$. Using the flow properties, the heat flux at the outer surface is found along with the local skin friction coefficients using the Eckert reference temperature method. With the thermal boundary conditions known, a predetermined number of thermal time steps are taken, each of size $\Delta t_{\mathrm{HT}}$, until the time $t_{0}+\Delta t_{\mathrm{AT}}$ is reached. The thermal loads based on the temperature change between $t_{0}$ and $t_{0}+\Delta t_{\mathrm{AE}}$ are then applied to the structural configuration at $t_{0}$. Additionally, the aerodynamic loads based on the already calculated flow properties are applied to the structure. The structural dynamic response solution is then marched forward one time step of size $\Delta t_{\mathrm{AE}}$. To march the lifting surface structural dynamic solution forward in time, the enforced displacements and accelerations at the interface due to fuselage motion must be specified. However, the fuselage motion in turn depends on the loads that the lifting surface exerts on the fuselage at the interface. Therefore, within each aeroelastic time step, iterations are performed between the lifting surface and fuselage, in which the lifting surface model passes interface loads to the fuselage, and the fuselage passes back interface motion. The mathematical details of how the iterations are performed are described in a subsequent section. Once the two components have been brought

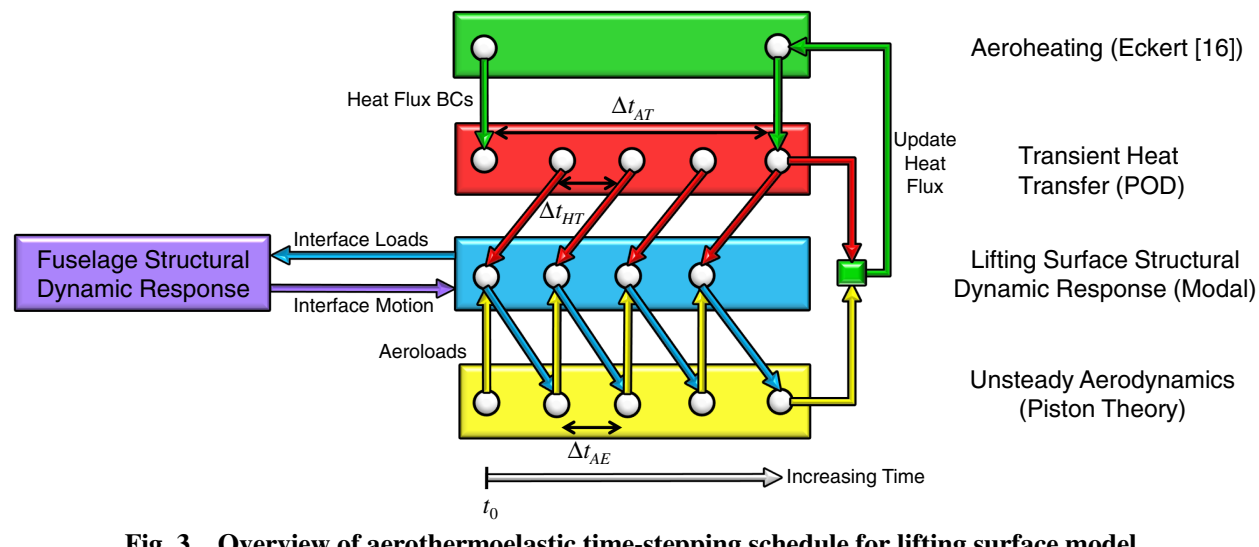

Fig. 3 Overview of aerothermoelastic time-stepping schedule for lifting surface model. 
into equilibrium at time $t_{0}+\Delta t_{\mathrm{AE}}$, the converged vector of lifting surface displacements is stored. These displacements are then fed back into the aerodynamic solver and the flow properties are calculated at time $t_{0}+\Delta t_{\mathrm{AE}}$ over the updated deformed configuration. The aeroelastic iterations continue to be carried out for a predetermined number of time steps. Once the time instant $t_{0}+\Delta t_{\mathrm{AT}}$ has been reached, the instantaneous flow properties and wall temperatures are used to update the heat flux boundary conditions to the thermal problem. With the updated thermal boundary conditions known, the transient thermal solution is marched forward from the time instant $t_{0}+\Delta t_{\mathrm{AT}}$ to the time instant $t_{0}+2 \Delta t_{\mathrm{AT}}$ and the process is repeated.

\section{Formulation of Equations of Motion}

\section{A. Fuselage Equations of Motion}

The equations of motion for the flexible hypersonic vehicle fuselage in three-dimensional (3-D) flight are derived using a Lagrangian approach. This approach is chosen instead of a Newtonian approach because it avoids the need to calculate internal forces within the structure. First, there are two frames of reference that must be introduced: an inertial Earth-fixed reference frame $(E$ frame) and a moving body-fixed reference frame ( $B$ frame). Next, the flight variables must be defined. The rigid-body translational velocity of the vehicle (i.e., the velocity of the $B$ frame origin with respect to the $E$ frame origin) is represented by $\beta$, and the rigid-body rotational velocity is represented by $\zeta$. The structural deformations are expressed in terms of the structural modal coordinates $\eta$. When the equations of motion are derived, it is assumed that the structural mode shapes are computed a priori. To derive the equations of motion using Lagrange's approach, the total kinetic and potential energies ( $T$ and $V$, respectively) are expressed in terms of the generalized coordinates and velocities $\eta, \beta$, and $\zeta$, as well as their time derivatives. Then, defining the Lagrangian as $\mathcal{L} \equiv T-V$, the equation of motion governing coordinate $q_{i}$ is given by

$$
\frac{\mathrm{d}}{\mathrm{d} t}\left(\frac{\partial \mathcal{L}}{\partial \dot{q}_{i}}\right)-\frac{\partial \mathcal{L}}{\partial q_{i}}=Q_{i}
$$

where $Q_{i}$ is the generalized force corresponding to $q_{i}$. The equations of motion can then be assembled into a matrix equation of the form

$$
\left[m_{f}\right]\left\{\begin{array}{l}
\dot{\beta} \\
\dot{\zeta} \\
\ddot{\eta}
\end{array}\right\}+\left[c_{f}\right]\left\{\begin{array}{l}
\beta \\
\zeta \\
\dot{\eta}
\end{array}\right\}+\left\{\begin{array}{c}
0 \\
0 \\
k_{f} \eta
\end{array}\right\}=\left\{\begin{array}{c}
Q_{\beta} \\
Q_{\zeta} \\
Q_{\eta}
\end{array}\right\}
$$

where $m_{f}$ is the generalized mass matrix, $c_{f}$ is the generalized damping matrix, and $k_{f}$ is the generalized stiffness matrix. The mass and damping are not constant (e.g., the mass matrix will change throughout flight as fuel is burned). The damping matrix, which contains the gyroscopic acceleration terms, is dependent on the rigidbody rotational velocity $\zeta$ and will also vary as the vehicle inertia changes. The details of this derivation of the equations of motion as applied to the free-free beam structure are available in [10].

Once the equations of motion are assembled, the next task is to determine the generalized forces $Q_{\beta}, Q_{\zeta}$, and $Q_{\eta}$. For the hypersonic vehicle fuselage, there are four sources of external loading: aerodynamics, propulsion, gravity, and resultant forces from the lifting surfaces, which include the lifting surfaces in this particular partition. Because the vehicle is undergoing unsteady rigid-body motion, as well as structural deflections, an unsteady aerodynamic model is required. The aerodynamic model used for the fuselage is based on a steady shock-expansion analysis with an unsteady correction computed using piston theory. These models are applied in a local inclination scheme [i.e., each panel of the outer mold line (OML) is considered separately, and the aerodynamic load on that panel is a function of its total velocity and orientation only].

The thrust required for hypersonic flight is provided by a scramjet engine. Many previous efforts in hypersonic vehicle simulation have relied on a simplified scramjet propulsion model [17], which uses quasi-1-D flow relations. An improved scramjet model [18] has been

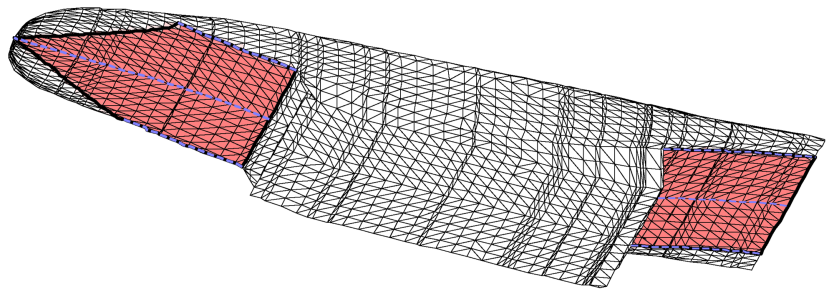

Fig. 4 Outer mold line panel kinematics.

recently made available. This model analyzes both the internal flowpath of the engine and the external inlet and nozzle. The external inlet and nozzle are analyzed with 2-D inviscid flow relations and include the effects of chemistry. The internal flowpath/combustor uses a quasi-1-D flow model that includes effects such as mixing. Because this overall model is essentially 2-D, it is applied to a 3-D vehicle in a strip-theory scheme. An example of how a scheme would be applied to a vehicle geometry is shown in Fig. 4, where the dashed lines represent the 2-D engine profiles that would be analyzed by the propulsion code. The 2-D profiles are determined by tracing from points at the edge of the engine cowl forward or backward along the OML. Although only three profiles are shown in Fig. $\underline{4}$, more may be used.

The effect of the lifting surfaces on the fuselage dynamics is expressed as a set of resultant forces and moments at the lifting surface attachment points. The resultant forces and moments are determined by passing the fuselage motion at the lifting surface attachment point to the lifting surface equations of motion. Using this specified root motion, the resultant forces and moments are calculated by the lifting surface aerothermoelastic model. These resultant forces include contributions from both the aerodynamic loading and the unsteady structural response of the lifting surfaces. As described in Sec. IV.A, the lifting surfaces are treated as allmovable surfaces about a hinge line located at the midchord. Deflection of the lifting surfaces by the control system is therefore represented as enforced rotation about this line.

\section{B. Lifting Surface Equations of Motion}

The usage of the Eckert reference temperature formulation used to calculate the aerodynamic heating over the lifting surface, the POD formulation for obtaining the transient temperature distribution of the lifting surface, and the third-order piston theory formulation for the lifting surface aerodynamic flow calculation are presented in a previous work [19] and are thus omitted here for brevity. Therefore, the emphasis of the current section is on the solution for the structural dynamic response of the lifting surface subjected to thermal and unsteady aerodynamic loads, as well as enforced displacements and accelerations due to fuselage motion.

For a lifting surface with prescribed accelerations and displacements at DOF $r$, the equations of motion can be partitioned as

$$
\begin{aligned}
& {\left[\begin{array}{ll}
M_{r r} & M_{r u} \\
M_{u r} & M_{u u}
\end{array}\right]\left\{\begin{array}{l}
\ddot{x}_{r} \\
\ddot{x}_{u}
\end{array}\right\}+\left[\begin{array}{ll}
K_{r r}^{*}(T) & K_{r u}^{*}(T) \\
K_{u r}^{*}(T) & K_{u u}^{*}(T)
\end{array}\right]\left\{\begin{array}{l}
x_{r} \\
x_{u}
\end{array}\right\}} \\
& =\left\{\begin{array}{c}
F_{r} \\
F_{u}^{H}+F_{u}^{A}
\end{array}\right\}
\end{aligned}
$$

where $M$ is the physical mass matrix, $x(t)$ are the physical degrees of freedom, $F^{H}(T)$ is the load vector due to heating, $F^{A}(t)$ is the load vector due to aerodynamic pressure, the subscript $r$ corresponds to the restrained DOF (those with prescribed accelerations and displacements), and the subscript $u$ corresponds to the unrestrained DOF (those without prescribed accelerations and displacements). The modified stiffness matrix $K^{*}(T)$ is given by

$$
K^{*}(T) \equiv K(T)+K_{G}(T)
$$

where $K(T)$ is the conventional stiffness matrix that varies due to the temperature dependence of the material properties, and $K_{G}(T)$ is the 
geometric stiffness matrix resulting from thermal stresses. In this formulation, the equations of motion for the unrestrained lifting surface DOF are cast in terms of the elastic displacements relative to the constraint motion caused by the enforced displacements at the restrained DOF. The term "constraint motion" refers to the displacements that the structure would undergo if the prescribed motion was applied statically and inertial effects were not present. Note that the term constraint motion is specifically used instead of "rigid-body motion" because the number of DOF with prescribed motion is greater than that required to constrain rigid-body motion in this case. Such a formulation is advantageous because the constraint motion is accounted for separately and the equations of motion are associated only with the elastic response, thus the structural modal matrix does not need to be modified to include constraint modes. Therefore, the lifting surface structural modal matrix is composed only of elastic modes in this formulation. The first step is to calculate the constraint motion due to enforced motion at the unrestrained DOF, denoted by $x_{u}^{C}$. This quantity is obtained by neglecting inertial loads and external loads in the second row of Eq. (3) and solving for $x_{u}$, that is,

$$
x_{u}^{C}=-\left(K_{u u}^{*}\right)^{-1} K_{u r}^{*} x_{r}
$$

Note that a transformation $U$ can be defined in Eq. (5) between $x_{u}^{C}$ and $x_{r}$ such that

$$
x_{u}^{C}=U x_{r}, \quad \text { where } U=-\left(K_{u u}^{*}\right)^{-1} K_{u r}^{*}
$$

If the number of DOF with prescribed motion were exactly equal to the minimum number of DOF required to constrain rigid-body motion, the columns of $U$ would represent rigid-body modes. Because in this case the number of DOF with prescribed motion is greater than that required to constrain rigid-body motion, the columns of $U$ represent constraint modes.

The next step is to derive the equations governing the elastic deformation of the unrestrained DOF $x_{u}^{E}$ relative to the constraint motion. Expanding Eq. (3), one obtains

$$
\begin{gathered}
M_{r r} \ddot{x}_{r}+M_{r u} \ddot{x}_{u}+K_{r r}^{*} x_{r}+K_{r u}^{*} x_{u}=F_{r} \\
M_{u r} \ddot{x}_{r}+M_{u u} \ddot{x}_{u}+K_{u r}^{*} x_{r}+K_{u u}^{*} x_{u}=F_{u}^{H}+F_{u}^{A}
\end{gathered}
$$

Recall that the total motion of the unrestrained DOF is the sum of the constraint motion plus the elastic motion, that is,

$$
x_{u}=x_{u}^{C}+x_{u}^{E}
$$

Substituting Eq. (8) into Eq. (7b), using Eq. (5) in the result, and bringing all terms associated with the restrained DOF to the righthand side results in

$$
\begin{aligned}
& M_{u u} \ddot{x}_{u}^{E}+K_{u u}^{*} x_{u}^{E}=-M_{u r} \ddot{x}_{r}+M_{u u}\left(K_{u u}^{*}\right)^{-1} K_{u r}^{*} \ddot{x}_{r}-K_{u r}^{*} x_{r} \\
& \quad+K_{u u}^{*}\left(K_{u u}^{*}\right)^{-1} K_{u r}^{*} x_{r}+F_{u}^{H}+F_{u}^{A}
\end{aligned}
$$

and simplifying the right-hand side of Eq. (9) gives

$$
M_{u u} \ddot{x}_{u}^{E}+K_{u u}^{*} x_{u}^{E}=\left[M_{u u}\left(K_{u u}^{*}\right)^{-1} K_{u r}^{*}-M_{u r}\right] \ddot{x}_{r}+F_{u}^{H}+F_{u}^{A}
$$

The relation given by Eq. (10) is the system to be solved for the relative elastic motion of the unrestrained DOF $x_{u}^{E}$. Note that the solution to Eq. (10) requires only the accelerations of the restrained DOF $\ddot{x}_{r}$ and not the displacements. However, $x_{r}$ is required to compute the constraint motion $x_{u}^{C}$. Because of the large number of degrees of freedom, direct solution of Eq. (10) within the aerothermoelastic lifting surface framework is not desirable. A common approach to reduce the order of such a system is to employ a modal transformation in which the structural displacements are expressed as a linear combination of a small number of basis vectors, which are the free vibration mode shapes of the structure. However, this approach cannot be applied directly for Eq. (10) because the mode shapes change over time due to modification of the stiffness from geometric stiffness and material degradation effects. The approach taken in this work is to first perform an offline calculation and select a reduced number of Ritz modes based on free vibration modes and load-dependent Ritz vectors [20] evaluated at a reference thermal state. These Ritz modes are then used as the modal basis for solution of the structural response throughout the simulation. This procedure is applicable because the Ritz modes need only to satisfy the geometric boundary conditions [21], which will always be the case regardless of the stiffness distribution. The modal matrix containing the structural reference modes $\Phi$ will not be updated throughout the simulation, thus preventing the need to solve an eigenvalue problem of the full system during the course of the simulation. Though the reference modes will not be updated throughout the simulation, the stiffness matrix will be updated each time the structural dynamic response is calculated to account for temperature-dependent material properties and geometric stiffening. Updating of the conventional stiffness matrix is performed using the temperature dependence of the material properties of the various materials. The geometric stiffness matrix is updated by solving a static finite element problem based on the thermal loads from temperatures at the current time step and the material coefficients of thermal expansion. As discussed previously, an important result of solving only for the elastic response in Eq. (10) is that the structural basis must only contain elastic modes. Because the remainder of the motion is accounted for in Eq. ( $\underline{8})$, the structural modal matrix need not contain constraint modes.

The reduced-order system is obtained by first representing the elastic motion $x_{u}^{E}(t)$ as a linear combination of the Ritz modes such that

$$
x_{u}^{E}(t)=\Phi d(t)
$$

where $d$ represents the modal coordinates of the Ritz modes that are stored as columns of the modal matrix $\Phi$. Note that, because the number of Ritz modes used in the modal expansion is much less than the number of physical degrees of freedom in the model, the computational cost of the solution is reduced. Once the modified stiffness matrix is known at the current time instant, the system is reduced by substituting Eq. (11) into Eq. (10) and premultiplying the system by $\Phi^{T}$ to project the system onto the basis, that is,

$$
\begin{aligned}
& \Phi^{T} M_{u u} \Phi \ddot{d}(t)+\Phi^{T} K_{u u}^{*}(T) \Phi d(t) \\
& =\Phi^{T}\left(\left[M_{u u}\left(K_{u u}^{*}(T)\right)^{-1} K_{u r}^{*}(T)-M_{u r}\right] \ddot{x}_{r}(t)+F_{u}(t, T)\right)
\end{aligned}
$$

where the net force $F_{u}(t, T)$ is defined as

$$
F_{u}(t, T) \equiv F_{u}^{H}(T)+F_{u}^{A}(t)
$$

The generalized mass matrix $m_{u u}$, generalized stiffness matrix $k_{u u}^{*}$, and generalized net force vector $f_{u}$ are then identified from Eq. ( $\left.\underline{12}\right)$ as

$$
m_{u u}=\Phi^{T} M_{u u} \Phi
$$

$$
k_{u u}^{*}(T)=\Phi^{T} K_{u u}^{*}(T) \Phi
$$

$$
f_{u}(t, T)=\Phi^{T} F_{u}(t, T)
$$

and the reduced system in modal form is given as

$$
\begin{aligned}
& m_{u u} \ddot{d}(t)+k_{u u}^{*}(T) d(t)=f_{u}(t, T) \\
& +\Phi^{T}\left(\left[M_{u u}\left(K_{u u}^{*}(T)\right)^{-1} K_{u r}^{*}(T)-M_{u r}\right] \ddot{x}_{r}(t)\right)
\end{aligned}
$$


Because the mass of the structure is taken to be constant in this work, the reference modes are orthogonal with respect to the mass matrix and the generalized mass matrix $m_{u u}$ reduces to the identity matrix. Because the modified stiffness matrix is continuously changing due to transient heating, we have no guarantee of orthogonality of the reference modes with respect to stiffness, and the equations are coupled. As such, the reduced-order system of equations in modal space is integrated numerically to calculate $d(t)$ at each aeroelastic time step. The numerical integration method employed is similar to the Newmark- $\beta$ method except that the load vector is averaged over three time instants and the stiffness matrix is modified such that the dynamic equation of motion reduces to a static solution if no inertial effects or damping exist [22]. The scheme uses a central finite difference representation for the velocity and acceleration at discrete times, given by [22]

$$
\begin{gathered}
\dot{d}^{(n)}=\frac{d^{(n+1)}-d^{(n-1)}}{2 \Delta t_{\mathrm{AE}}} \\
\ddot{d}^{(n)}=\frac{d^{(n+1)}-2 d^{(n)}+d^{(n-1)}}{\Delta t_{\mathrm{AE}}^{2}}
\end{gathered}
$$

where the superscript $(n)$ refers to the time level. The initial conditions $d^{(0)}$ and $\dot{d}^{(0)}$ are used to generate the vectors $d^{(n-1)}, f_{u}^{(n-1)}$, and $f_{u}^{(n)}$ for the initial time step $n=0$ using

$$
\begin{gathered}
d^{(-1)}=d^{(0)}-\dot{d}^{(0)} \Delta t_{\mathrm{AE}} \\
f_{u}^{(-1)}=k_{u u}^{*} d^{(-1)} \\
f_{u}^{(0)}=k_{u u}^{*} d^{(0)}
\end{gathered}
$$

Note that this formulation assumes that the initial acceleration for all points is zero (initial velocity is constant). To maintain consistency with the central difference approximation for the modal accelerations, the enforced acceleration in Eq. (15) $\ddot{x}_{r}(t)$ is approximated at time level $(n)$ using a central difference formula, that is,

$$
\ddot{x}_{r}(t)=\frac{x_{r}^{(n+1)}-2 x_{r}^{(n)}+x_{r}^{(n-1)}}{\Delta t_{\mathrm{AE}}^{2}}
$$

Substituting the finite difference approximations of the velocities and accelerations, Eqs. (16) and (18), into the equations of motion (15) and averaging the applied loads over three adjacent time instants, the equations of motion are rewritten as

$$
H_{1} d^{(n+1)}=H_{2}+H_{3} d^{(n)}+H_{4} d^{(n-1)}
$$

where

$$
H_{1}=\frac{1}{\Delta t_{\mathrm{AE}}^{2}} m_{u u}+\frac{1}{3} k_{u u}^{*}
$$

$$
\begin{aligned}
& H_{2}=\frac{1}{3}\left(f_{u}^{(n+1)}+f_{u}^{(n)}+f_{u}^{(n-1)}\right) \\
& +\Phi^{T}\left[M_{u u}\left(K_{u u}^{*}(T)\right)^{-1} K_{u r}^{*}(T)-M_{u r}\right] \\
& \times \frac{x_{r}^{(n+1)}-2 x_{r}^{(n)}+x_{r}^{(n-1)}}{\Delta t_{\mathrm{AE}}^{2}}
\end{aligned}
$$

$$
\begin{aligned}
& H_{3}=\frac{2}{\Delta t_{\mathrm{AE}}^{2}} m_{u u}-\frac{1}{3} k_{u u}^{*} \\
& H_{4}=\frac{-1}{\Delta t_{\mathrm{AE}}^{2}} m_{u u}-\frac{1}{3} k_{u u}^{*}
\end{aligned}
$$

The vector of structural modal coordinates at the end of the time step $d^{(n+1)}$ is obtained by decomposing $H_{1}$ and applying it to the righthand side of Eq. (19). Once $d^{(n+1)}$ is obtained, the total motion of the unconstrained degrees of freedom in physical space is computed via Eqs. (ㅁ),$(\underline{8})$, and (11) using

$$
x_{u}^{(n+1)}=-\left(K_{u u}^{*}\right)^{-1} K_{u r}^{*} x_{r}^{(n+1)}+\Phi d^{(n+1)}
$$

Once the displacements of the unrestrained DOF are known at a given time $t$, the force contribution due to lifting surface (wing) motion $F_{r}^{W}$ can be calculated at time $t$ by computing the quantity $M_{r u} \ddot{x}_{u}+K_{r u}^{*} x_{u}$ from the first row of Eq. (3) and moving it to the right-hand side to treat as a forcing function acting on the fuselage at the interface. This force contribution is computed using

$$
\begin{aligned}
& F_{r}^{W}(t, T)=-M_{r u} \frac{x_{u}^{(n+1)}-2 x_{u}^{(n)}+x_{u}^{(n-1)}}{\Delta t_{\mathrm{AE}}^{2}} \\
& -K_{r u}^{*} \frac{x_{u}^{(n+1)}+x_{u}^{(n)}+x_{u}^{(n-1)}}{3}
\end{aligned}
$$

where the accelerations of the unrestrained DOF $\ddot{x}_{u}$ are calculated using a central difference and the displacements are averaged over three adjacent time levels to maintain consistency with the numerical integration scheme. Once $F_{r}^{W}(t, T)$ is known, it can then be passed to the fuselage equations of motion to update the loads. To compute the net external force that the lifting surface (wing) exerts relative to the fuselage (body) $F_{r}^{W / B}$, the elastic motion of the unrestrained lifting surface DOF relative to the interface DOF is used. Therefore, $F_{r}^{W / B}$ is given by

$$
F_{r}^{W / B}=-K_{r u}^{*}\left(x_{u}^{E}-x_{u, T}^{E}\right)
$$

where $x_{u, T}^{E}$ is the elastic deformation caused by thermal loads. Note that $x_{u, T}^{E}$ must be subtracted from $x_{u}^{E}$ because thermal loads are internal to the system and do not result in external forces being exerted on the fuselage.

\section{Methodology for Fuselage-Lifting Surface Coupling}

As described previously, the flight dynamics simulation framework for the HSV is based on a partitioned approach in which the vehicle substructures are modeled independently and interface information is exchanged between substructures within each time step. The example case considered here focuses on the coupling of a hypersonic vehicle all-movable lifting surface and fuselage. The equations of motion for the fuselage and the lifting surface are presented earlier, and thus the final step of the formulation is to couple the two systems. Referring to Eq. (3), the fuselage motion at the lifting surface-fuselage interface is given by

$$
M_{r r} \ddot{x}_{r}(t)+K_{r r} x_{r}(t)=F_{r}+F_{r}^{W}(t, T)
$$

where $M_{r r}$ and $K_{r r}$ are the mass and stiffness associated with the interface DOF, $x_{r}(t)$ is the degree of freedom corresponding to the fuselage displacement, and $F_{r}^{W}(t, T)$ is the force exerted by the lifting surface on the fuselage given in Eq. (22). The force component $F_{r}$ represents the force due to the weight of the fuselage and is given by

$$
F_{r}=M_{f} g
$$

where $g$ is the gravitational acceleration. For uniformity, the same numerical integration scheme used for the lifting surface is also used 
for the fuselage, such that the fuselage displacement at the end of each aeroelastic time step $(n+1)$ can be found from

$$
I_{1} x_{r}^{(n+1)}=I_{2}+I_{3} x_{r}^{(n)}+I_{4} x_{r}^{(n-1)}
$$

where

$$
\begin{gathered}
I_{1}=\frac{1}{\Delta t_{\mathrm{AE}}^{2}} M_{r r}+\frac{1}{3} K_{r r} \\
I_{2}=\frac{1}{3}\left(F_{r}^{(n+1)}+F_{r}^{(n)}+F_{r}^{(n-1)}\right)+F_{r}^{W} \\
I_{3}=\frac{2}{\Delta t_{\mathrm{AE}}^{2}} M_{r r}-\frac{1}{3} K_{r r} \\
I_{4}=\frac{-1}{\Delta t_{\mathrm{AE}}^{2}} M_{r r}-\frac{1}{3} K_{r r}
\end{gathered}
$$

Note that $F_{r}^{W}$ is already averaged over three adjacent time steps in Eq. (22), and therefore only $F_{r}$ must be averaged in Eq. (27b).

The effect of the lifting surface response on the fuselage response can be seen by inspecting Eq. (26). Specifically, note that $I_{2}$ is a function of $F_{r}^{W}$, and $F_{r}^{W}$ depends on $x_{u}^{(n+1)}$. Also, recall that $x_{u}^{(n+1)}$ is expressed in terms of the lifting surface modal coordinates $d^{(n+1)}$. As shown in Eq. $(20 \mathrm{~b}), d^{(n+1)}$ in turn depends on $x_{r}^{(n+1)}$. We can therefore state that $d^{(n+1)}$ is a function of $x_{r}^{(n+1)}$. To highlight these dependencies, we rewrite Eq. (26) as

$$
I_{1} x_{r}^{(n+1)}=I_{2}\left(d^{(n+1)}\left(x_{r}^{(n+1)}\right)\right)+I_{3} x_{r}^{(n)}+I_{4} x_{r}^{(n-1)}
$$

Solution of Eq. (28) is complicated due to the fact that $d^{(n+1)}$ is unknown, and depends on the solution to the fuselage response. In a manner analogous to Eqs. (19) and (28) for the lifting surface can be rewritten as

$$
H_{1} d^{(n+1)}=H_{2}\left(x_{r}^{(n+1)}\left(d^{(n+1)}\right)\right)+H_{3} d^{(n)}+H_{4} d^{(n-1)}
$$

where the dependence of $\mathrm{H}_{2}$ on both the lifting surface and fuselage states at time $(n+1)$ is explicitly shown.

Although both the fuselage and lifting surface systems could be combined into one [as shown in Eq. (3)] and solved monolithically, the goal of this work is to develop a methodology for time marching multiple coupled systems of differential equations by treating each independently and exchanging interface information in a mathematically robust manner. The motivation for such an approach is because the HSV will consist of multiple components, each of which will have its own model. Because the models are likely to be of dissimilar form, direct monolithic coupling of the models is not easily facilitated. The philosophy of this work is therefore to treat each model as a black box with respect to the other. Mathematically, this means that $I_{2}\left(d^{(n+1)}\left(x_{r}^{(n+1)}\right)\right)$ in Eq. $(\underline{28})$ and $H_{2}\left(x_{r}^{(n+1)}\left(d^{(n+1)}\right)\right)$ in Eq. (29) are treated as unknown functions of $x_{r}^{(n+1)}$ and $d^{(n+1)}$, where information about these functions can only be obtained by evaluating them at specific input values.

Because of the unknown nature of $H_{2}$ and $I_{2}$, Eqs. (28) and (29) are each treated as if they are nonlinear in the other system's states (i.e., the fuselage system is treated as if it is nonlinear in the lifting surface states, and the lifting surface system is treated as if it is nonlinear in the fuselage states). Such an assumption preserves the generality of the method such that it is applicable to any two systems of differential equations that are nonlinearly coupled. Furthermore, the methodology is formulated such that, if the two systems are only linearly coupled, the linear solution is recovered in only one iteration.
Because of the assumption of nonlinear coupling, the equations are solved using an iterative approach to bring the two systems to equilibrium at the end of each time step. As opposed to a pseudotime-marching strategy in which the two components are successively marched in pseudotime until the equilibrium state is reached, this work formulates the algorithm as a solution to a nonlinear root-finding problem and uses derivative information of the nonlinear function to converge to the equilibrium state more efficiently. A secant method is used in this work to increment the state at each iteration due to its property of being superlinearly locally convergent [23].

Before implementing the methodology, it must be decided which system will be the driving system and which system will be the driven system. The driving system is the one in which the secant method is used to compute the increment to the state at each iteration until convergence is achieved. The state vector for the driven system at time $(n+1)$ is merely updated by marching forward one step based on the state vector from the driving system at time $(n+1)$ for each iteration. Assuming that the driving system is chosen to be the fuselage system, we begin by moving all terms in Eq. (28) to the lefthand side and equating it to the residual at iteration $k$, $R\left(d_{k}^{(n+1)}\left(x_{r, k}^{(n+1)}\right)\right)$, that is,

$$
\begin{aligned}
& I_{1} x_{r, k}^{(n+1)}-I_{2}\left(d_{k}^{(n+1)}\left(x_{r, k}^{(n+1)}\right)\right)-I_{3} x_{r}^{(n)}-I_{4} x_{r}^{(n-1)} \\
& \quad=R\left(d_{k}^{(n+1)}\left(x_{r, k}^{(n+1)}\right)\right)
\end{aligned}
$$

Note that, according to Eq. (28), the right-hand side of Eq. (30) should be equal to zero. However, this condition is only achieved when the lifting surface and fuselage are brought into equilibrium at the end of the time step $(n+1)$. At intermediate iterations before reaching the equilibrium state, the left-hand side of Eq. (30) will be equal to a nonzero residual that is a function of the fuselage and lifting surface states. The process of bringing the lifting surface and fuselage to equilibrium is mathematically equivalent to driving the residual to zero in Eq. (30) by iterating on the fuselage state vector $x_{r}^{(n+1)}$.

A summary of the algorithm used to bring the fuselage and lifting surface systems to equilibrium within each time step is given in Table 1 . The iteration procedure begins with two initial guesses to the fuselage state at the end of the time step, $x_{r, 0}^{(n+1)}$ and $x_{r, 1}^{(n+1)}$, where the subscripts zero and one refer to the iteration number. For each initial guess, $H_{2}$ is computed at step 3 using Eq. (20b), and Eq. (19) is solved

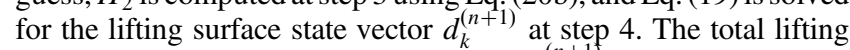
surface displacements in physical space $x_{u}^{(n+1)}$ are then computed using Eq. (21) in step 5. Once the motion of the lifting surface at time level $(n+1)$ is known, the loads imparted on the fuselage by the lifting surface at time level $(n+1)$ are calculated using Eq. (22) to give $F_{r}^{W}(t, T)$ in step 6 . With these loads known, $I_{2}$ is computed from Eq. (27b). Using $I_{2}$, the left-hand side of Eq. (30) is evaluated giving the residual at step 8 . At this point, if the residual is above the specified tolerance, an iteration loop begins in which the fuselage state is incremented using the fuselage states and residual values at the two previous iterations via the secant method. Iterations are carried out repeating the preceding steps $3-8$, until the residual drops below the specified tolerance. At that point, the fuselage and lifting surface have been brought into equilibrium at the end of the current time step. The states for each component are then stored, the simulation marches to the next time step, and the iteration process is again carried out until equilibrium is achieved at the next time step. This process continues until the simulation is complete.

A schematic of the information flow for the iteration scheme is given in Fig. 5 . The process begins by calculating the increment to the fuselage state at the interface and updating it at the next iteration. Based on the new interface displacement, the accelerations of the DOF at the lifting surface attachment point are found using a finite difference formula. These accelerations are then passed to lifting surface equations of motion. Using these boundary conditions, the lifting surface state vector at the next iteration is computed by marching from time $(n)$ to time $(n+1)$. Once the solution of the lifting surface equations of motion is known, the loads exerted by the lifting surface on the fuselage are computed using a finite difference 
Table 1 Iteration scheme used for bringing lifting surface and fuselage into equilibrium at each time step

\begin{tabular}{ll}
\hline \hline Step number & Instruction \\
\hline 1$)$ & $x_{r, 0}^{(n+1)}, x_{r, 1}^{(n+1)}=$ initial guesses \\
$2)$ & for $k=0,1$ \\
$3)$ & $H_{2}=1 / 3\left(f_{u}^{(n+1)}+f_{u}^{(n)}+f_{u}^{(n-1)}\right)+\Phi^{T}\left[M_{u u}\left(K_{u u}^{*}(T)\right)^{-1} K_{u r}^{*}(T)-M_{u r}\right]\left(x_{r, k}^{(n+1)}-2 x_{r}^{(n)}+x_{r}^{(n-1)} / \Delta t_{\mathrm{AE}}^{2}\right)$ \\
$4)$ & $d_{k}^{(n+1)}=H_{1}^{-1}\left(H_{2}+H_{3} d^{(n)}+H_{4} d^{(n-1)}\right)$ \\
$5)$ & $x_{u}^{(n+1)}=-\left(K_{u u}^{*}\right)^{-1} K_{u r}^{*} x_{r}^{(n+1)}+\Phi d_{k}^{(n+1)}$ \\
$6)$ & $F_{r}^{W}(t, T)=-M_{r u}\left(x_{u}^{(n+1)}-2 x_{u}^{(n)}+x_{u}^{(n-1)} / \Delta t_{\mathrm{AE}}^{2}\right)-K_{r u}^{*}\left(x_{u}^{(n+1)}+x_{u}^{(n)}+x_{u}^{(n-1)} / 3\right)$ \\
$7)$ & $I_{2}=1 / 3\left(F_{r}^{(n+1)}+F_{r}^{(n)}+F_{r}^{(n-1)}\right)+F_{r}^{W}$ \\
$8)$ & $R_{k}=I_{1} x_{r, k}^{(n+1)}-I_{2}\left(d_{k}^{(n+1)}\left(x_{r, k}^{(n+1)}\right)\right)-I_{3} x_{r}^{(n)}-I_{4} x_{r}^{(n-1)}$ \\
$9)$ & end \\
$10)$ & while $\left|R_{1}\right|>\operatorname{tol}^{1}{ }_{\mathrm{a}}$ \\
$11)$ & $x_{r, 2}^{(n+1)}=x_{r, 1}^{(n+1)}-R_{1}\left(x_{r, 1}^{(n+1)}-x_{r, 0}^{(n+1)}\right) / R_{1}-R_{0}$ \\
$12)$ & $H_{2}=1 / 3\left(f_{u}^{(n+1)}+f_{u}^{(n)}+f_{u}^{(n-1)}\right)+\Phi^{T}\left[M_{u u}\left(K_{u u}^{*}(T)\right)^{-1} K_{u r}^{*}(T)-M_{u r}\right]\left(x_{r, 2}^{(n+1)}-2 x_{r}^{(n)}+x_{r}^{(n-1)} / \Delta t_{\mathrm{AE}}^{2}\right)$ \\
$13)$ & $d_{2}^{(n+1)}=H_{1}^{-1}\left(H_{2}+H_{3} d^{(n)}+H_{4} d^{(n-1)}\right)$ \\
$14)$ & $x_{u}^{(n+1)}=-\left(K_{u u}^{*}\right)^{-1} K_{u r}^{*} x_{r}^{(n+1)}+\Phi d_{2}^{(n+1)}$ \\
$15)$ & $F_{r}^{W}(t, T)=-M_{r u}\left(x_{u}^{(n+1)}-2 x_{u}^{(n)}+x_{u}^{(n-1)} / \Delta t_{\mathrm{AE}}^{2}\right)-K_{r u}^{*}\left(x_{u}^{(n+1)}+x_{u}^{(n)}+x_{u}^{(n-1)} / 3\right)$ \\
$16)$ & $I_{2}=1 / 3\left(F_{r}^{(n+1)}+F_{r}^{(n)}+F_{r}^{(n-1)}\right)+F_{r}^{W}$ \\
$17)$ & $R_{2}=I_{1} x_{r, 2}^{(n+1)}-I_{2}\left(d_{2}^{(n+1)}\left(x_{r, 2}^{(n+1)}\right)\right)-I_{3} x_{r}^{(n)}-I_{4} x_{r}^{(n-1)}$ \\
$18)$ & $x_{r, 0}=x_{r, 1}$ \\
$19)$ & $x_{r, 1}=x_{r, 2}$ \\
$20)$ & $R_{0}=R_{1}$ \\
$21)$ & $R_{1}=R_{2}$ \\
$22)$ & end \\
\hline
\end{tabular}

${ }^{a}$ tol = convergence tolerance

formula and passed back to the fuselage system. The fuselage state is again incremented, except this time using the new loads from the lifting surface. At this point, the process repeats and iterations are carried out until the forces exerted by the lifting surface on the fuselage are brought into equilibrium with the accelerations of the fuselage at the interface location.

\section{Fuselage-Lifting Surface Configuration}

With the equations of motion for the HSV fuselage and lifting surface presented and the partitioned solution scheme outlined, the current section describes a simplified fuselage-lifting surface configuration to which the methodology is applied. The vehicle of interest for this work is shown in Fig. 6. This specific geometry was provided by Vibroacoustics Solutions, Inc. The OML includes both the fuselage as well as the scramjet cowl. The recessed region on the top of the fuselage accommodates a spacecraft used for launch purposes. The lifting surfaces at the aft section of the vehicle are all-movable lifting surfaces, which rotate about a hinge line located at their midchord. These surfaces are not considered part of the fuselage structure.

Although the fuselage and lifting surface shown in Fig. 6 collectively represent the overall HSV geometry of interest, this work is specifically interested in demonstrating the partitioned solution methodology used to couple these two structures. As such, a simplified configuration to be used in this work to exemplify the methodology is highlighted. The simplified configuration consists of the all-movable lifting surface attached to a single-degree-of-freedom oscillator, which replaces the fuselage. The use of this configuration will allow for a focus on the partitioned solution methodology and the effects of fuselage-lifting surface inertial coupling, while still retaining the essential physics of the problem.

\section{A. Lifting Surface Model}

A finite element model representing the all-movable lifting surfaces depicted in Fig. 6 has been created for use in this study. The thickness from the top skin layer to the bottom skin layer is $4 \%$ chord length [25]. The top and bottom skin layers are each equipped with two $3.8-\mathrm{mm}$-thick thermal protection system layers, and thus the thickness of the outer mold line is $4 \%$ chord length plus the $15.2 \mathrm{~mm}$ of thermal protection system material. The chord length at the root is $5.2 \mathrm{~m}(17 \mathrm{ft})$ [26] and the leading edge makes an angle of $34 \mathrm{deg}$ with the $y$ axis, whereas the trailing edge makes an angle of $18 \mathrm{deg}$ with the $y$ axis [27]. Planform and cross-sectional views of the lifting surface are given in Figs. 7 and 8, respectively. The finite element model of the lifting surface is shown in Fig. 9 with the top surface removed for visualization purposes. Details associated with the materials selection, material properties, thermal protection system, and finite element model are presented in a previous paper by the authors [14] and are thus omitted here.

\section{B. Simplified Fuselage-Lifting Surface Configuration}

This work is motivated by two main objectives. The first is to develop and validate a partitioned solution methodology for coupling the various substructures of the HSV for time-domain flight dynamic simulation. The second is to use the partitioned solution methodology to assess the impact of lifting surface inertial effects on overall vehicle response. To focus specifically on these objectives, the fuselage is replaced by a simplified representation for the purposes of this study. This representation consists of a point mass attached to a spring, which is then connected to the lifting surface. The lifting surface and single-degree-of-freedom oscillator configuration is depicted in Fig. 10. Note that the motion of the fuselage mass is constrained such that it is only permitted to translate in the $z$ direction. Therefore, the enforced motion at the lifting surface attachment location only contains a $z$ translational component. The attachment between the fuselage mass and the lifting surface is modeled by a rigid element (Nastran RBE2) that transfers the fuselage motion to the lifting surface DOF located approximately within the attachment region 


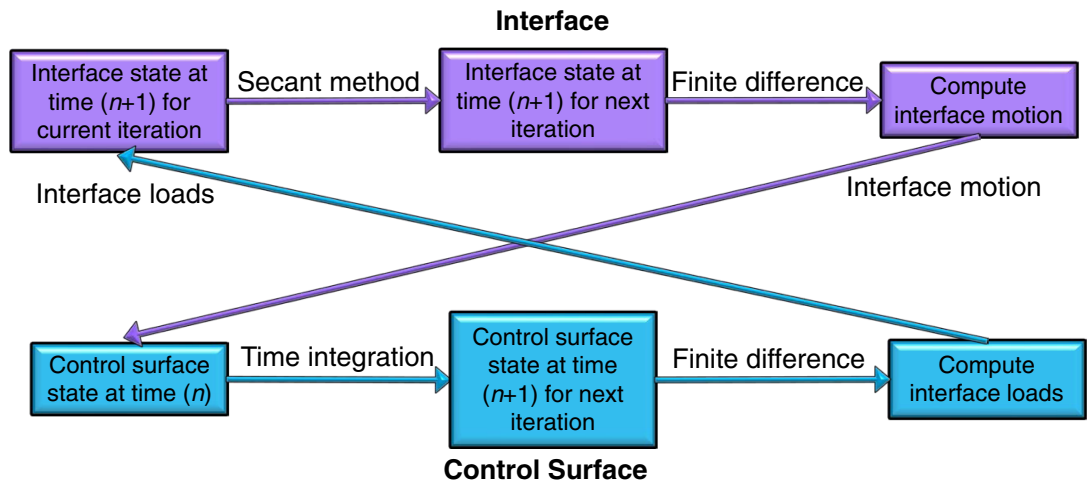

Fig. 5 Schematic of fuselage-lifting surface coupling scheme.

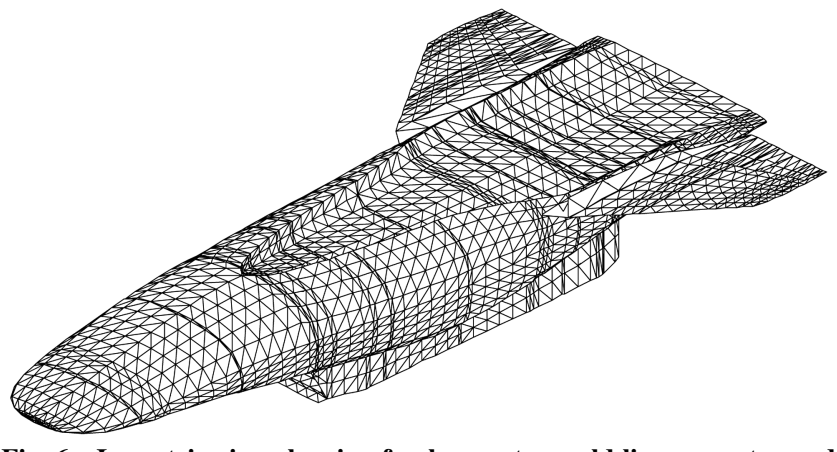

Fig. 6 Isometric view showing fuselage outer mold line geometry and location of lifting surfaces.

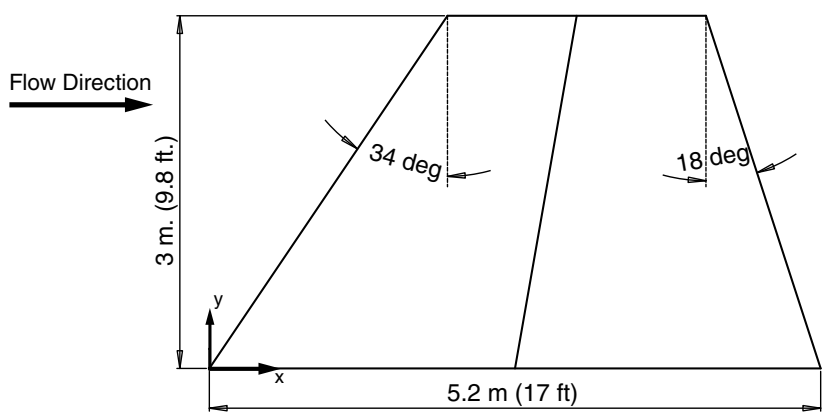

Fig. 7 Planform geometry of lifting surface model.

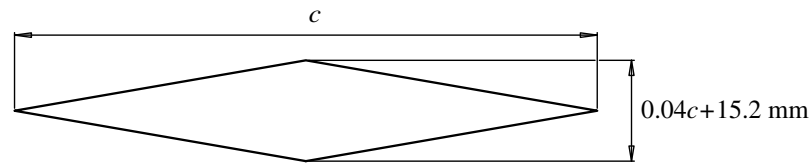

Fig. 8 Cross-sectional geometry of lifting surface model.

shown in Fig. 9. Physically, the spring stiffness $K_{f}$ represents the bending stiffness of the fuselage as well as the associated aerodynamic stiffness. The goal is to march the complete system in time by modeling the lifting surface and fuselage (point mass) independently and exchanging information between the two within each time step. Although the partitioned solution methodology is applied to the configuration of Fig. 10 in this paper, it is important to note that the approach is general in nature such that it can be applied to any configuration of interest, including the HSV representation shown in Fig. 6 .

\section{Aerothermoelastic Lifting Surface ROM Generation}

Before carrying out simulations, the thermal and structural dynamic ROMs to be used in the lifting surface framework must be created. As some of the ROMs used in the lifting surface

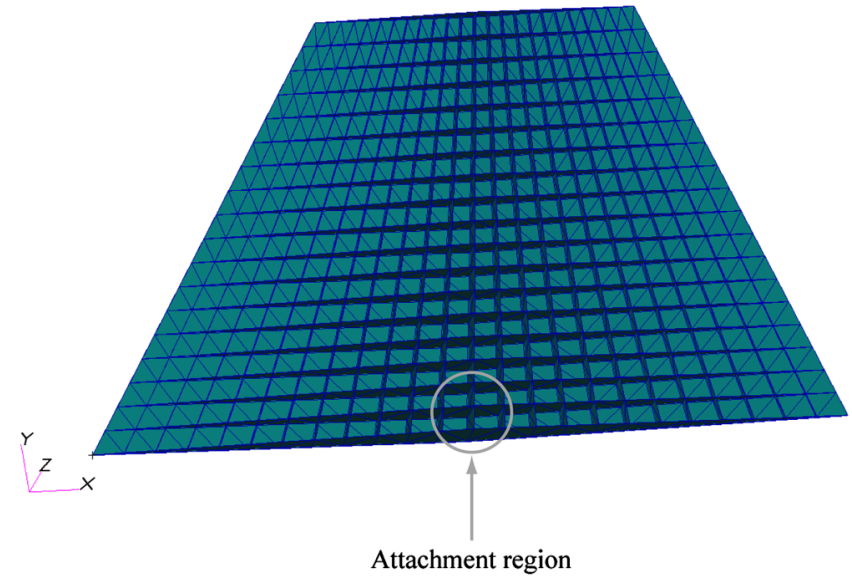

Fig. 9 Finite element model of lifting surface used in study.

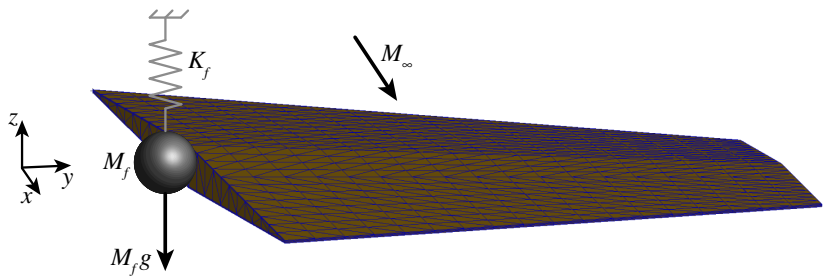

Fig. 10 Representation of lifting surface and fuselage system.

aerothermoelastic simulation process are dependent on components of other ROMs, these models must be generated in a specific order. An overview of the ROM process is given in [15] and is thus omitted here for brevity.

The aerothermoelastic lifting surface ROM used in the current work is based on that created in a previous work [15]. The ranges in flight conditions used in ROM generation for that work are given in Table 2 , where $M_{\infty}$ is the freestream Mach number, $\alpha_{\text {net }}$ is the net lifting surface angle of attack, $h$ is the altitude, and $\mathcal{T}_{0}$ is the uniform initial temperature condition. The thermal snapshots were based on 10 parallel aerothermoelastic simulations, resulting in a total of 49,510 thermal snapshots. Based on the POD eigenvalues, 32 POD modes were retained for the thermal ROM. Comparison of the thermal ROM to the full-order thermal model for one representative

Table 2 Bounds on flight conditions for aerothermoelastic lifting surface ROM

\begin{tabular}{lcc}
\hline \hline Lower bound & Parameter & Upper bound \\
\hline 5.0 & $\leq M_{\infty} \leq$ & 8.0 \\
$0.0 \mathrm{deg}$ & $\leq \alpha_{\text {net }} \leq$ & $4.0 \mathrm{deg}$ \\
25.0 & $\leq h \leq$ & $45.0 \mathrm{~km}$ \\
293 & $\leq \mathcal{T}_{0} \leq$ & $1500 \mathrm{~K}$ \\
\hline \hline
\end{tabular}


Table 3 Parameters used in time dependence of enforced motion at lifting surface attachment point

\begin{tabular}{ccc}
\hline \hline$i$ & $\omega_{i}(\mathrm{rad} / \mathrm{s}) / f_{i} \mathrm{~Hz}$ & $\mu_{i}$ \\
\hline 1 & $6.9 / 1.1$ & 0.015 \\
2 & $43.2 / 6.9$ & 0.015 \\
3 & $121.1 / 19.3$ & 0.015 \\
\hline \hline
\end{tabular}

simulation showed that the $L_{\infty}$ error remained below $3 \%$ throughout the complete time range considered [15].

Although the current work makes use of the thermal ROM that was generated in [15], additional studies are conducted in this paper to identify a robust structural basis under thermal, aerodynamic, and base excitation loads. For the purpose of ROM assessment, the time dependence of the enforced displacements in the $z$ direction at the lifting surface attachment point $w_{a}(t)$ is prescribed as

$$
w_{a}(t)=\sum_{i=1}^{3} \mu_{i} \sin \left(\omega_{i} t\right)
$$

where $\mu_{i}$ are real scalar coefficients and $\omega_{i}$ are circular frequencies representing the first three fuselage bending natural frequencies. These frequencies are computed based on vehicle properties given in a previous work [10]. Table $\underline{3}$ gives the circular natural frequencies along with the corresponding values of the ordinary natural frequencies $f_{i}$ in hertz as well as the scalar coefficients.

To determine the appropriate number of free vibration modes and load-dependent Ritz vectors to include in the structural basis, fulland reduced-order simulations are performed under combined base excitation loads, thermal loads, and unsteady aerodynamic loads at the following flight conditions: $M_{\infty}=6.5, \alpha_{\text {net }}=2 \mathrm{deg}$, and $h=35 \mathrm{~km}$. The thermal load vector used in the simulations is held constant over time and is obtained based on the temperatures at the end of a $400 \mathrm{~s}$ aerothermal simulation beginning from room temperature at these flight conditions. By including both base excitation loads and thermal loads in the simulations, the ability of the basis to capture both highly unsteady response due to base motion as well as static response due to thermal deformation can be examined. Based on simulation results, the structural basis is chosen to consist of 10 free vibration modes evaluated at the reference thermal state and five additional load-dependent Ritz vectors. The reference thermal state is obtained by averaging the 49,510 thermal snapshots taken in [15]. Of the 10 free vibration modes employed in the basis, the natural frequency of the lowest frequency mode is $25 \mathrm{~Hz}$, whereas that of the highest frequency mode is $186 \mathrm{~Hz}$. Each of the five load-dependent Ritz vectors correspond to a static solution under a representative applied load. The applied load for each Ritz vector is chosen based on a POD analysis of the structural load vector, which was carried out in [15] to identify the dominant spatial components of the structural loads.

Recall that two additional approximations are made in the structural dynamic ROM besides the use of the modal subset. One involves the kriging approximations used to directly update the generalized stiffness matrix $k_{u u}^{*}(T)$ and physical thermal load vector $F_{u}^{H}(T)$ in Eqs. (12) and (13) as a function of temperature. The other involves the fact that $K_{u u}^{*}(T)$ and $K_{u r}^{*}(T)$ on the right-hand sides of Eqs. (5) and (12) are evaluated at the reference thermal state and held constant throughout the simulation. To distinguish the errors due to these approximations from the errors due to modal truncation, three different cases are simulated, as shown in Table 4.
In the table, "actual $T$ " indicates that a quantity is evaluated at the actual thermal state of the structure, whereas "ref. $T$ " indicates that the quantity is evaluated at the reference thermal state obtained by averaging the thermal snapshots. The term "exact" in the table refers to the fact that no approximation is made when assembling the matrix or vector, whereas "kriging" indicates that the corresponding kriging approximation is used. For case E1, the full-order structural model is used and all quantities are evaluated exactly at the actual thermal state. The full-order model does not require the generalized stiffness matrix as indicated by "N/A" in the third column for case E1. For this case, the physical stiffness matrix $K_{u u}^{*}$ on the left-hand side of Eq. (10) is evaluated exactly at the actual thermal state. Case E2 uses the structural ROM with all quantities evaluated exactly at the actual thermal state. The structural ROM is also used for case E3 and $k_{u u}^{*}(T)$ and $F_{u}^{H}(T)$ are again evaluated at the actual thermal state. However, for this case, these quantities are computed using the corresponding kriging approximations, which provide improved computational efficiency. Additionally, for case E3, $K_{u u}^{*}(T)$ and $K_{u r}^{*}(T)$ on the righthand sides of Eqs. (5) and (12) are evaluated at the reference thermal state and held constant throughout the simulation. Therefore, the difference between cases E1 and E2 will demonstrate the error incurred solely due to the use of the modal subset in the structural ROM. The difference between cases E2 and E3 will illustrate the error incurred solely due to the kriging approximations of the generalized stiffness and physical thermal loads in addition to that due to evaluating $K_{u u}^{*}(T)$ and $K_{u r}^{*}(T)$ on the right-hand sides of Eqs. (5) and (12) at the reference thermal state instead of the actual thermal state. Note that case E3 represents the structural dynamic model that will be used in the remainder in the paper.

Plots of the $z$-direction displacements from cases E1-E3 for a duration of $1 \mathrm{~s}$ are given at four different nodes in Fig. 11. Though there exist minor discrepancies at certain peaks, the results given in Fig. 11 provide evidence that the structural dynamic lifting surface ROM represented by case E3 is capable of capturing the full-order response represented by case $\mathrm{E} 1$ with reasonable accuracy. Therefore, the 15-mode structural dynamic ROM of the lifting surface as described earlier is used in the remainder of this study.

A related paper by the authors [28] gives further validation of the structural dynamic ROM as well as validation of the partitioned solution against a monolithic solution. That discussion is omitted from this paper for brevity. However, it should be noted that only one iteration is required for convergence of the partitioned solution for the case configuration considered in this paper. This is because $R$ is a linear function of $x_{r}^{(n+1)}$ for the configuration employed here. Thus, once $R$ is evaluated at the two initial guesses generated in step 1 of Table 1 , the slope of $R$ is known and the converged value of $x_{r}^{(n+1)}$ can be obtained in the first iteration of the secant method. However, the methodology is intentionally formulated to be general such that the problem need not be linear. If the problem is in fact linear, then the linear solution is recovered in the minimum number of iterations.

\section{Lifting Surface-Fuselage Coupling: Results}

\section{A. Impact of Lifting Surface Inertia on Fuselage $z$-Direction Re-} sponse

With the lifting surface ROM validated, the partitioned solution is now used to assess the impact of lifting surface inertial loads. To assess these effects, the coupled fuselage-lifting surface formulation described previously is used to perform simulations of the system depicted in Fig. 10 in which lifting surface inertial effects are both present and absent. For the case in which lifting surface inertial effects are present, the coupled formulation described previously, which includes lifting surface structural dynamics, is employed. For

Table 4 Summary of three cases used in structural dynamic ROM evaluation

\begin{tabular}{lccccc}
\hline \hline Case & Structural Model & $k_{u u}^{*}(T)$ & $F_{u}^{H}(T)$ & $K_{u u}^{*}(T)$ & $K_{u r}^{*}(T)$ \\
\hline E1 & Full-order & N/A & Actual $T /$ exact & Actual $T /$ exact & Actual $T /$ exact \\
E2 & 15-mode ROM & Actual $T /$ exact & Actual $T /$ exact & Actual $T /$ exact & Actual $T /$ exact \\
E3 & 15-mode ROM & Actual $T /$ kriging & Actual $T /$ kriging & Ref. $T /$ exact & Ref. $T /$ exact \\
\hline \hline
\end{tabular}




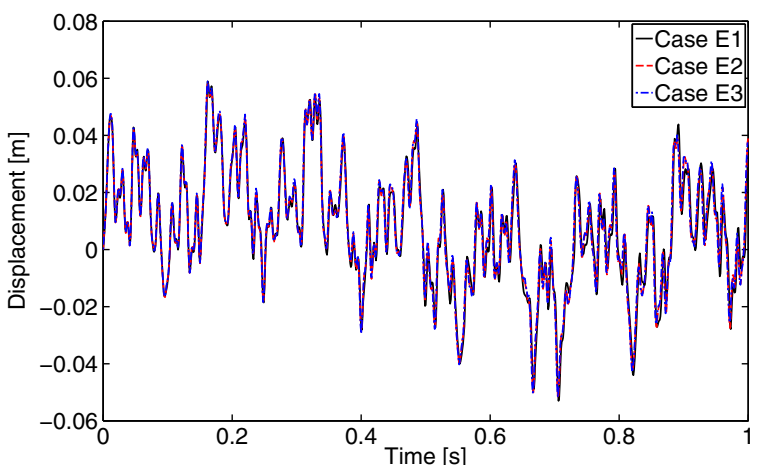

a) Node 2 (bottom surface, root leading edge)

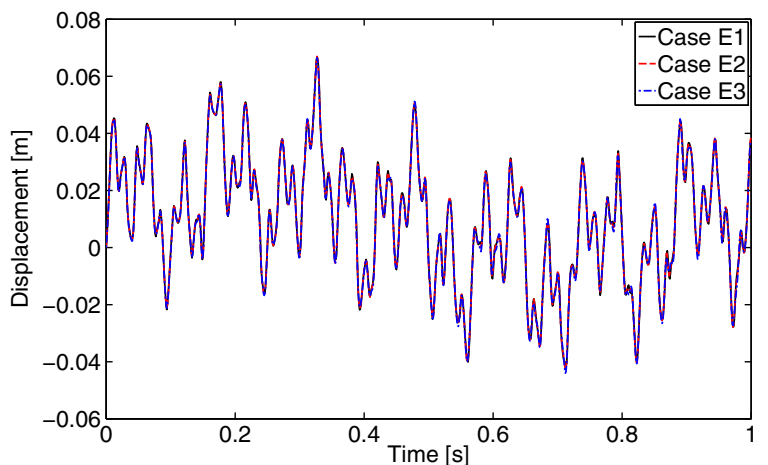

c) Node 458 (bottom surface, root trailing edge)

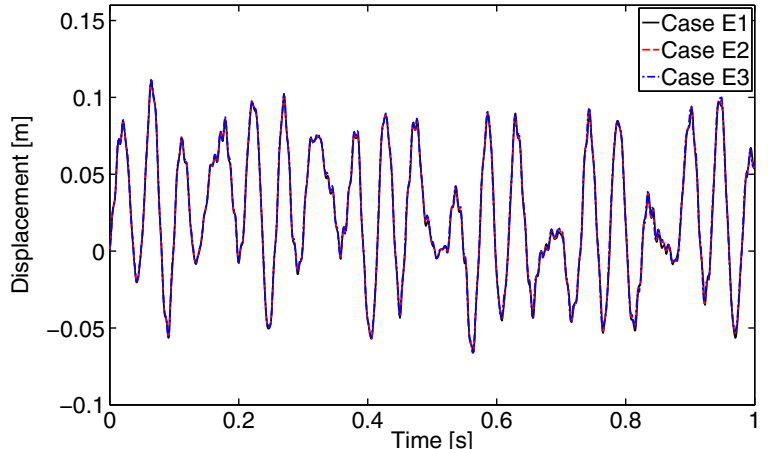

b) Node 37 (bottom surface, tip leading edge)

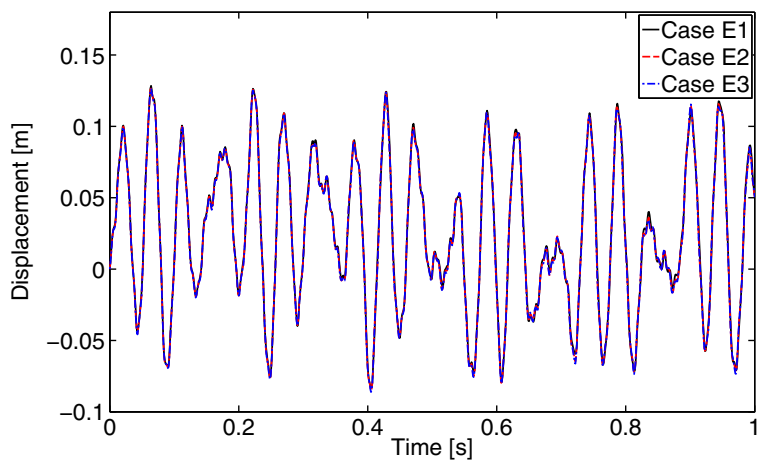

d) Node 475 (bottom surface, tip trailing edge)

Fig. 11 Time history of $z$ displacements at four selected nodes for cases E1-E3.

the case in which lifting surface inertial effects are absent, the coupled formulation is modified such that the solution for the lifting surface deformation is reduced to the solution of a static problem subjected to thermal and aerodynamic loads with the constraint motion imposed instantaneously. Elimination of lifting surface inertial effects is achieved by modifying Eq. (7b) to include only static terms such that it becomes

$$
K_{u r}^{*} x_{r}+K_{u u}^{*} x_{u}=F_{u}^{H}+F_{u}^{A}
$$

To maintain consistency with the dynamic lifting surface solution, Eq. (32) is solved in a manner analogous to that derived in Eqs. (815). Therefore, for the static lifting surface case, the equations governing the lifting surface modal coordinates are reduced to

$$
k_{u u}^{*}(T) d(t)=f_{u}(t, T)
$$

The net external force exerted by the lifting surface relative to the fuselage $F_{r}^{W / B}$ is again calculated using Eq. (23) for the static lifting surface case. However, for the static lifting surface case, $x_{u}^{E}$ will not include elastic deformation due to lifting surface inertia and will thus allow for assessment of the impact of lifting surface inertia on the loads that the lifting surface exerts on the fuselage.

Before performing simulations, $M_{f}$ is adjusted such that the fuselage weight is exactly balanced by the $z$-direction force produced by the lifting surface at $M_{\infty}=6.5, \alpha_{\text {net }}=2 \mathrm{deg}$, and $h=35 \mathrm{~km}$. Additionally, the spring stiffness associated with the fuselage is set such that the fuselage natural frequency is $10 \mathrm{~Hz}$ in the absence of lifting surface inertia. All simulations in this section begin by heating the lifting surface for $400 \mathrm{~s}$ at $M_{\infty}=6.5, \alpha_{\text {net }}=2 \mathrm{deg}$, and $h=$ $35 \mathrm{~km}$ and bringing it to elastic equilibrium under thermal and aerodynamic loads. As the weight of the fuselage is equal to the $z$ force produced by the lifting surface under these conditions, the fuselage will be at its equilibrium position at the beginning of the simulation. The simulation is then started at these flight conditions by time marching the partitioned lifting surface-fuselage formulation forward in the manner described previously. For all subsequent results in this work, the time step sizes are chosen as follows: $\Delta t_{\mathrm{AE}}=\Delta t_{\mathrm{HT}}=0.001 \mathrm{~s}$ and $\Delta t_{\mathrm{AT}}=0.1 \mathrm{~s}$.

Though the thermal and aerodynamic loads will change over time as the simulation progresses, the impact of these loads on the response of the system is minimal because they change on a slow timescale. Thus, it is necessary to perturb the equilibrium conditions such that structural dynamic oscillations are induced into the system so that the impact of inertial effects can be examined. The method used here to perturb the equilibrium is to apply a change in flow direction for a short period of time, which corresponds to a change in lifting surface angle of attack $\alpha_{\text {net }}$. The flow direction is instantaneously incremented at time $t_{i}$ by an amount $\Delta \alpha_{\text {net }}$, held it at this value for a time duration $\Delta t_{\alpha}$, and then decremented back to the original value of $\alpha_{\text {net }}$ at time $t_{d}=t_{i}+\Delta t_{\alpha}$. The results presented in this paper use $\Delta \alpha_{\text {net }}=8 \mathrm{deg}$, which corresponds to increasing $\alpha_{\text {net }}$ from 2 to $10 \mathrm{deg}$ at $t_{i}$, holding at $10 \mathrm{deg}$ for a time duration of $\Delta t_{\alpha}$, and then decrementing back to 2 deg at $t_{d}$. The values of $\Delta t_{\alpha}$ that are used in the simulations are 0.03 and $0.1 \mathrm{~s}$. Note that, for the results presented, $\Delta \alpha_{\text {net }}$ is applied at $0.01 \mathrm{~s}$ into the transient $\left(t_{i}=0.01 \mathrm{~s}\right)$.

The displacements of the mass representing the fuselage are shown in Fig. 12 for a $1 \mathrm{~s}$ time history, where "CS dynamic" refers to the case that uses the structural dynamic solution to obtain the lifting surface response at each aeroelastic time step, whereas "CS static" refers to the case that uses the static solution to obtain the lifting surface response at each aeroelastic time step. Figure 12a shows results for the case of $\Delta t_{\alpha}=0.03 \mathrm{~s}$, whereas Fig. $12 \mathrm{~b}$ shows results for the case of $\Delta t_{\alpha}=0.1 \mathrm{~s}$. For $\Delta t_{\alpha}=0.03 \mathrm{~s}, \alpha_{\text {net }}$ is decremented back from 10 to 2 deg just before the fuselage reaches the peak of its response. However, for $\Delta t_{\alpha}=0.1 \mathrm{~s}, \alpha_{\text {net }}$ is not decremented back from 10 to 2 deg until the fuselage has approximately returned its equilibrium position. Therefore, comparing Fig. 12a with Fig. 12b, it can be seen that the fuselage undergoes significantly higher amplitude oscillations for the case of $\Delta t_{\alpha}=0.03 \mathrm{~s}$ than for the case of $\Delta t_{\alpha}=0.1 \mathrm{~s}$. Comparing the CS dynamic and CS static cases in Fig. 12a, one can observe a difference in frequency of oscillation resulting from the inclusion of lifting surface inertia. Recall that the mass and stiffness properties associated with the fuselage were set such that its natural frequency would be $10 \mathrm{~Hz}$ in the absence of 


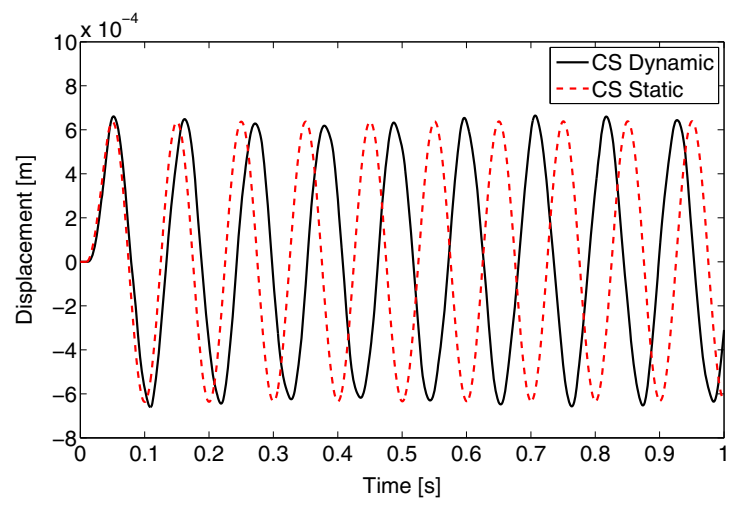

a) $\Delta t_{\alpha}=0.03 \mathrm{~s}$

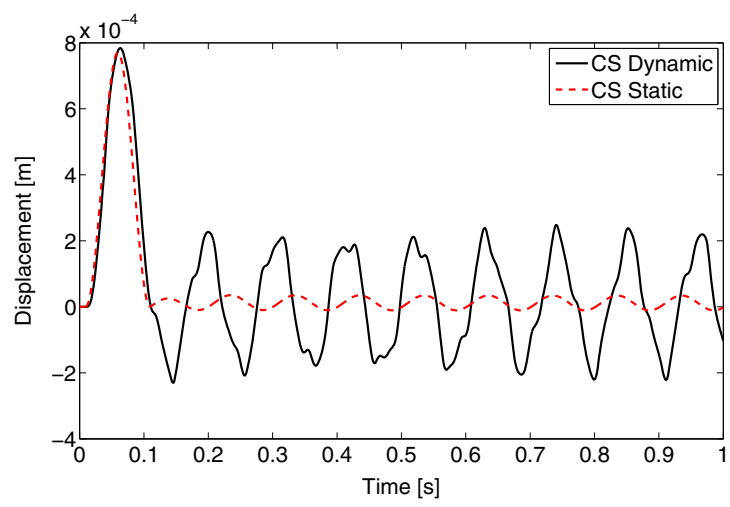

b) $\Delta t_{\alpha}=0.1 \mathrm{~s}$

Fig. 12 Fuselage displacements for two values of $\Delta t_{\alpha}$ with and without lifting surface inertial effects.

lifting surface inertia. Examining the CS static case in Fig. 12a, this $10 \mathrm{~Hz}$ natural frequency is observed. However, by including lifting surface inertia, the frequency of fuselage oscillation is reduced, as shown by the CS dynamic case in Fig. 12a. Note that, for the case of $\Delta t_{\alpha}=0.03 \mathrm{~s}$, lifting surface inertia has only a minor effect on the amplitude of fuselage oscillation for the CS dynamic case. Examining Fig. $12 \mathrm{~b}$, it can be seen that, when $\Delta t_{\alpha}$ is increased to $0.1 \mathrm{~s}$, lifting surface inertia has a more prominent effect on the amplitude of the fuselage response. This is because $\alpha_{\text {net }}$ is decremented back to $2 \mathrm{deg}$ approximately at the same time that the fuselage reaches its equilibrium position. Thus, for the CS static case, the amplitude of fuselage oscillation is reduced significantly. The result is that the forces acting on the fuselage due to its own inertia and stiffness are smaller compared with the lifting surface inertial loads beyond $t_{d}$. Therefore, lifting surface inertia has a more prominent effect on fuselage amplitude of oscillation beyond $t_{d}$ for this case.

To examine the frequency content of the fuselage response, the fast Fourier transform (FFT) is performed on the fuselage response for the time history beginning at $t_{d}$. Because the FFT assumes that the input sequence is a power of two in length [23], the FFT is performed on a signal in the time range $0.041 \leq t \leq 1.064 \mathrm{~s}$ for $\Delta t_{\alpha}=0.03 \mathrm{~s}$ and in the range $0.1110 .111 \leq t \leq 1.134 \mathrm{~s}$ for $\Delta t_{\alpha}=0.1 \mathrm{~s}$. Therefore, the output signal is of length 1024 for both values of $\Delta t_{\alpha}$. The singlesided amplitude spectrums of the fuselage displacements are given in Figs. $13 \mathrm{a}$ and $13 \mathrm{~b}$ for the cases of $\Delta t_{\alpha}=0.03$ and $\Delta t_{\alpha}=0.1 \mathrm{~s}$, respectively. For both values of $\Delta t_{\alpha}$, the first peak at nonzero frequency occurs at approximately $8.79 \mathrm{~Hz}$ for the CS dynamic case and at approximately $9.77 \mathrm{~Hz}$ for the CS static case, indicating that exclusion of lifting surface inertia results in an $11 \%$ overprediction in the lowest frequency fuselage oscillation component. In addition to the $10 \mathrm{~Hz}$ mode, results from the CS dynamic case show higher frequency oscillation components associated with lifting surface modes. For both values of $\Delta t_{\alpha}$, the second and third major peaks

\begin{tabular}{lc}
$\begin{array}{c}\text { Table } 5 \\
\text { First } \mathbf{1 0} \text { lifting surface } \\
\text { natural frequencies evaluated }\end{array}$ \\
thermal state obtained by heating \\
structure for $\mathbf{4 0 0}$ s at $\boldsymbol{M}_{\infty}=\mathbf{6 . 5}$, \\
$\boldsymbol{\alpha}_{\text {net }}=\mathbf{2}$ deg, and $\boldsymbol{h}=\mathbf{3 5} \mathbf{~ k m}$ \\
\hline \hline Mode no. & Natural frequency, Hz \\
\hline 1 & 25.1 \\
2 & 35.6 \\
3 & 53.2 \\
4 & 54.3 \\
5 & 86.1 \\
6 & 98.1 \\
7 & 130 \\
8 & 144 \\
9 & 163 \\
10 & 183 \\
\hline \hline
\end{tabular}

occur at approximately 26.4 and $54.7 \mathrm{~Hz}$, respectively. To determine which lifting surface modes these frequencies correspond to, the first 10 lifting surface natural frequencies are computed at the actual thermal state of the structure, which is obtained by heating the lifting surface for $400 \mathrm{~s}$ at the previously described flight conditions. These first 10 natural frequencies are given in Table 5. Based on the frequencies shown in the table, it can be concluded that the second major peaks for the CS dynamic cases in both Figs. 13a and 13b correspond to the first lifting surface mode, whereas the third major peaks correspond to either the third or fourth lifting surface mode. It should be noted that a previous work [15] described a mode switching phenomenon that was found to occur between lifting surface modes three and four as the structure is heated. This phenomenon is likely because these modes are close in frequency. Though there are three noticeable peaks present in the FFT results of the fuselage displacements, the lowest frequency mode clearly contributes most significantly to the response for both values of $\Delta t_{\alpha}$.

Plots of the lifting surface $z$ displacements at node 247 (located on the bottom surface at the midchord of the tip) are shown in Figs. 14a and $14 \mathrm{~b}$ for $\Delta t_{\alpha}=0.03$ and $\Delta t_{\alpha}=0.1 \mathrm{~s}$, respectively. For the $\overline{\mathrm{CS}}$ static case in both figures, there is an instantaneous jump in displacement at $t_{i}=0.01 \mathrm{~s}$. This is due to the increase in aerodynamic pressure loads on the structure induced by the increased angle of attack. Because the lifting surface response is calculated using a static solution for the CS static cases, the increase in displacement is instantaneous for these cases. Beyond $t_{d}$, the lifting surface motion in the CS static case is approximately the same as the fuselage motion with an offset due to static deformation under thermal and aerodynamic loads. Note that, although the thermal and aerodynamic loads do change over time in the static case, their effect on lifting surface deformation is essentially a static effect because these loads change on a slow timescale. Comparing the CS dynamic cases to the CS static cases, it can be observed that sustained structural dynamic oscillations are induced in the lifting surface, which lead to higher displacement levels than would be predicted if only a static lifting surface solution was used.

The FFTs of the lifting surface response at node 247 are taken for the same time periods as used in generating Figs. 13a and 13b. The single-sided amplitude spectrums for the two values of $\Delta t_{\alpha} \overline{\text { are given }}$ in Figs. $15 \mathrm{a}$ and $15 \mathrm{~b}$. For both values of $\Delta t_{\alpha}$, the first major peak at nonzero frequency occurs at approximately $8.79 \mathrm{~Hz}$ in the CS dynamic case and at approximately $9.77 \mathrm{~Hz}$ in the CS static case. For the CS dynamic case, the second major peak occurs at approximately $26.4 \mathrm{~Hz}$ for both values of $\Delta t_{\alpha}$. The frequencies of the first two lifting surface peaks are the same as those for the first two fuselage peaks. Although these first two peaks contribute most significantly to the lifting surface response for the CS dynamic case, there exist three additional smaller peaks for both values of $\Delta t_{\alpha}$, which occur at approximately $35.2,54.7$, and $83.0 \mathrm{~Hz}$. Referring to Table 5, these three peaks correspond roughly with lifting surface modes two, three/ four, and five, respectively. Comparing the lifting surface FFT results with those of the fuselage, it can be seen that, whereas lifting surface 


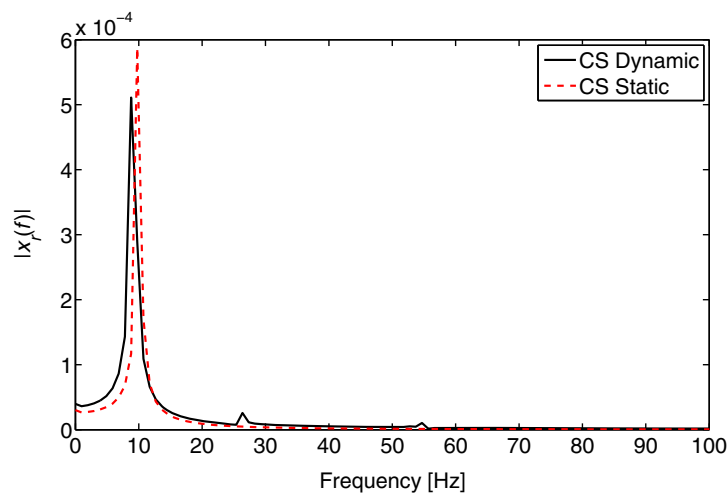

a) $\Delta t_{\alpha}=\mathbf{0 . 0 3} \mathrm{s}$

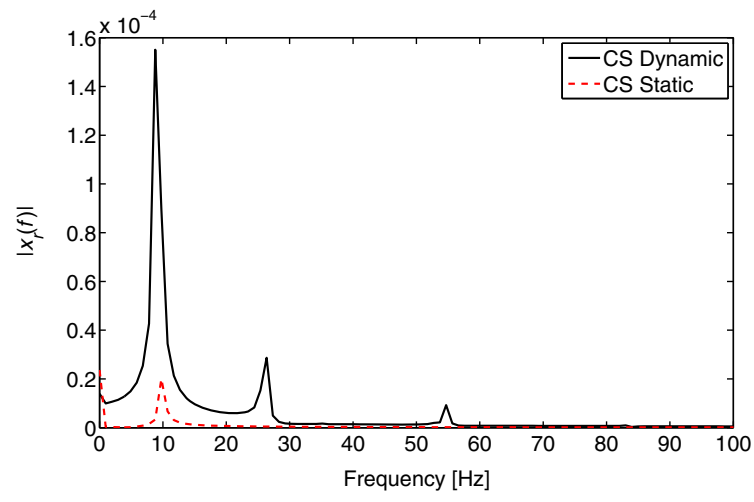

b) $\Delta t_{\alpha}=0.1 \mathrm{~s}$

Fig. 13 FFT of fuselage response for two values of $\Delta t_{\alpha}$ with and without lifting surface inertial effects.

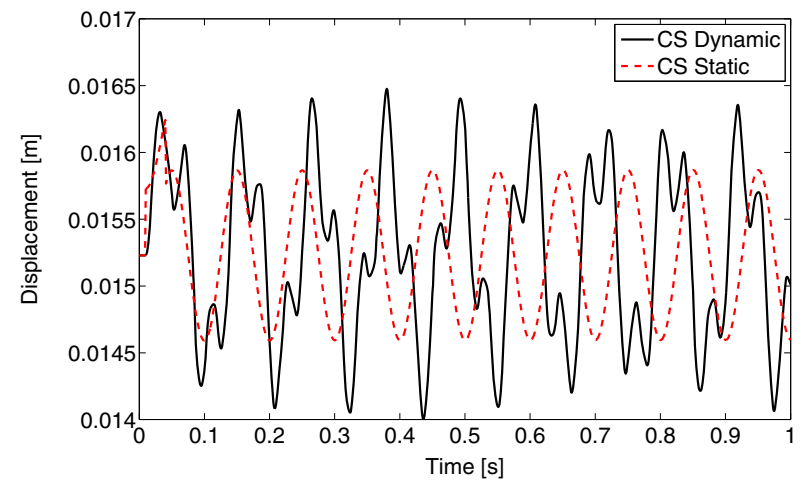

a) $\Delta t_{\alpha}=\mathbf{0 . 0 3} \mathrm{s}$

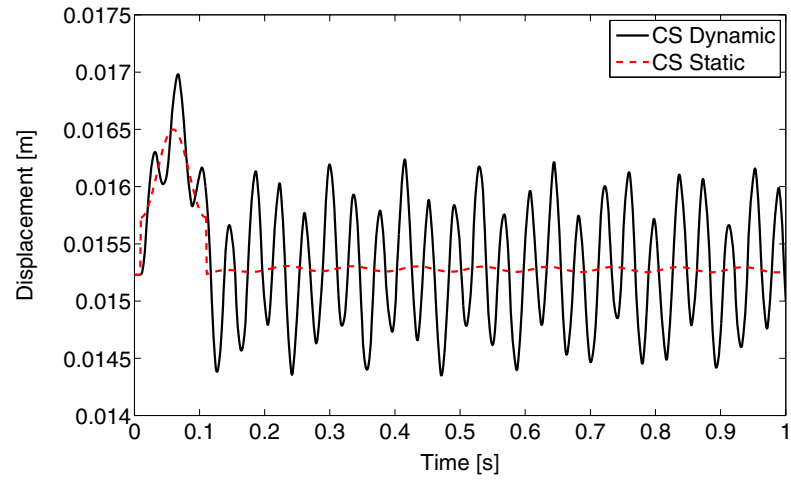

b) $\Delta t_{\alpha}=0.1 \mathrm{~s}$

Fig. 14 Lifting surface $z$ displacements at node 247 (bottom surface, tip, midchord) for two values of $\Delta t_{\alpha}$ with and without lifting surface inertial effects.

modes $1-5$ are all excited at least to a small extent in the lifting surface response, only lifting surface modes one and three/four appear to have an effect on the fuselage response. However, as was the case for the fuselage, the two lowest frequency modes contribute most significantly to the lifting surface response.

The loads exerted by the lifting surface on the fuselage $F_{r}^{W / B}$ are shown in Fig. 16 for the two values of $\Delta t_{\alpha}$. As expected, in the CS static cases, $F_{r}^{\overline{W / B}}$ is approximately constant with an instantaneous increase at $t_{i}$ and instantaneous decrease at $t_{d}$ due to the change in $\alpha_{\text {net }}$. Examining the CS dynamic results, $F_{r}^{W / B}$ is shown to oscillate approximately about the static value due to the lifting surface inertial effects. As shown by the fuselage displacements in Fig. 12, the extent to which the fuselage responds to these high-frequency oscillations in $F_{r}^{W / B}$ depends upon the inertia and stiffness of the fuselage itself.
Based on the results presented for this time history, the maximum absolute ratio of $F_{r}^{W / B}$ for the dynamic case to that for the static case is seven for $\Delta t_{\alpha}=0.03 \mathrm{~s}$ and eight for $\Delta t_{\alpha}=0.1 \mathrm{~s}$. These results indicate that lifting surface inertia can have a noticeable effect on fuselage loads. However, the extent to which lifting surface inertial loads impact the fuselage response is dependent on the fuselage inertia as well as the frequency content of the lifting surface inertia loads.

Plots of the FFT of the $F_{r}^{W / B}$ time history for the two values of $\Delta t_{\alpha}$ are given in Figs. 17a and 17b. In both plots, noticeable peaks occur for the CS dynamic case at approximately 8.79, 26.4, 52.7, 54.7, and $84.0 \mathrm{~Hz}$. Although the modes at these frequencies each contribute to $F_{r}^{W / B}$ at least to a small extent, the modes corresponding to the first four of these frequencies contribute most significantly. Referring to Table 5, these four frequencies roughly correspond to fuselage mode
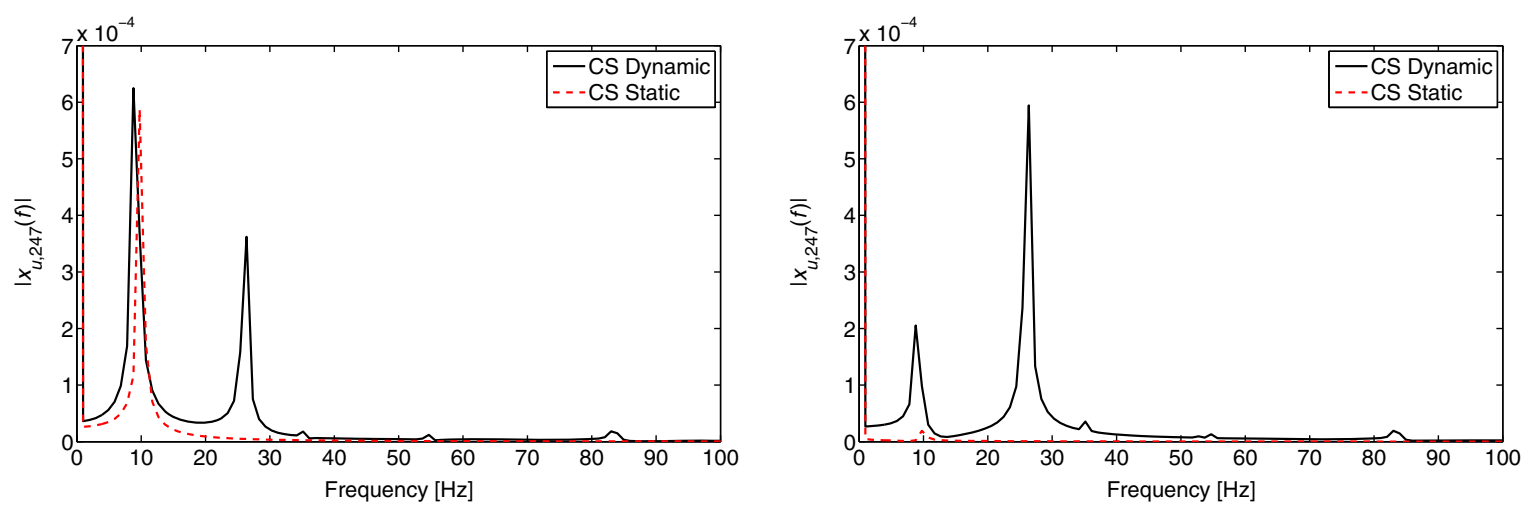

a) $\Delta t_{\alpha}=0.03 \mathrm{~s}$

b) $\Delta t_{\alpha}=0.1 \mathrm{~s}$

Fig. 15 FFT of lifting surface response at node 247 (bottom surface, tip, midchord) for two values of $\Delta t_{\alpha}$ with and without lifting surface inertial effects. 


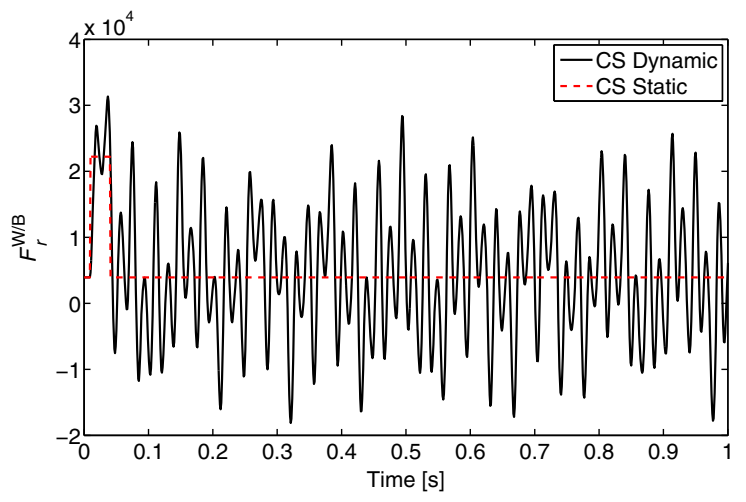

a) $\Delta t_{\alpha}=\mathbf{0 . 0 3} \mathrm{s}$

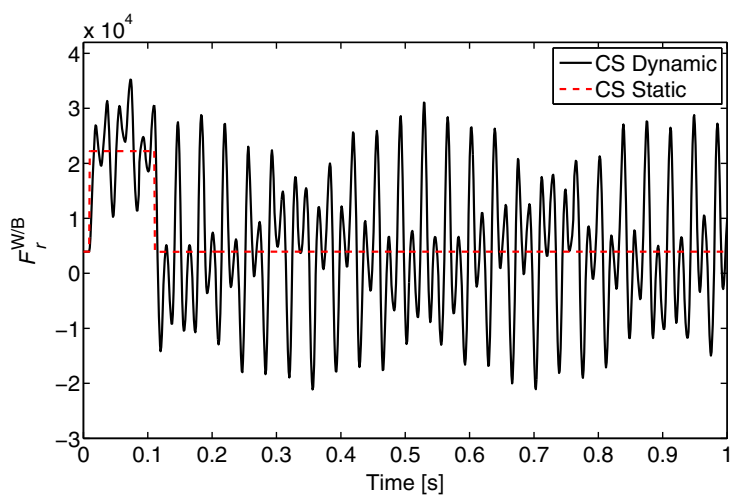

b) $\Delta t_{\alpha}=0.1 \mathrm{~s}$

Fig. $16 F_{r}^{W / B}$ for two values of $\Delta t_{\alpha}$ with and without lifting surface inertial effects.

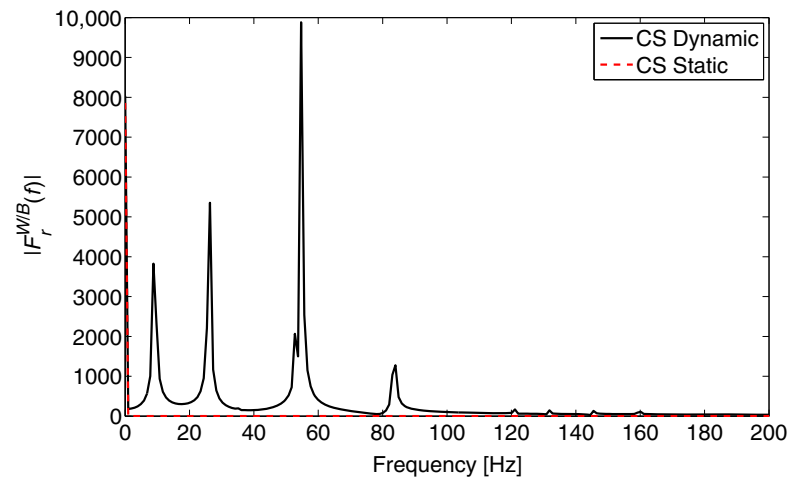

a) $\Delta t_{\alpha}=0.03 \mathrm{~s}$

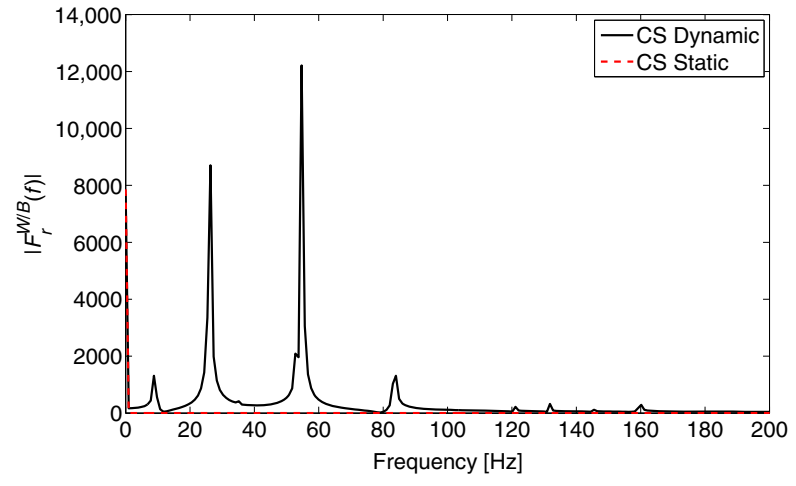

b) $\Delta t_{\alpha}=0.1 \mathrm{~s}$

Fig. 17 FFT of $F_{r}^{W / B}$ response for two values of $\Delta t_{\alpha}$ with and without lifting surface inertial effects.

1, lifting surface mode 1 , lifting surface mode 3 , and lifting surface mode 4 . The most dominant contribution to $F_{r}^{W / B}$ comes from the $54.7 \mathrm{~Hz}$ mode for both values of $\Delta t_{\alpha}$. However, it is interesting to note that the $54.7 \mathrm{~Hz}$ mode is not the most dominant mode in either the fuselage response or lifting surface response.

\section{B. Impact of Lifting Surface Inertia Under Commanded Change in Deflection Angle}

The next aspect of this study involves investigation of the impact of lifting surface inertial loads under a commanded change in lifting surface deflection angle imposed via the control system. The importance of understanding such lifting surface-fuselage inertial coupling has been discussed in a recent paper [9], which referred to this coupling as the "tail-wags-dog" effect. The discussion in [9] highlights the fact that this effect typically results in a complexconjugate pair of zeros in the elevator-to-pitch rate transfer function and can affect the speed of response of the system.

As a step toward assessing the extent to which the overall vehicle pitch response is affected by lifting surface inertia under commanded changes in deflection angle, a control input corresponding to lifting surface deflection angle is incorporated into the aerothermoelastic ROM framework. Note that all simulations conducted in this section do not include the fuselage mass depicted in Fig. 10 and consist of enforced lifting surface motion due to rotation about the hinge line. To capture the relationship between the input command from the controller and the resulting output rotation applied to the lifting surface, actuator dynamics are incorporated into the lifting surface model. The equation relating the input command $\delta_{\text {cmd }}$ to the output lifting surface deflection angle $\delta$ is given by [29]

$$
\ddot{\delta}=-2 \zeta_{\delta} \omega_{\delta} \dot{\delta}-\omega_{\delta}^{2} \delta+\omega_{\delta} \delta_{\text {cmd }}
$$

where $\zeta_{\delta}=1$ and $\omega_{\delta}=20$. A schematic illustrating the geometry associated with the lifting surface deflection angle is given in Fig. 18, where $x_{B}$ and $z_{B}$ represent the body-fixed axis system. As shown in Fig. $18, \delta$ is taken to be positive leading edge up. Note that the net lifting surface angle of attack $\alpha_{\text {net }}$ is given by

$$
\alpha_{\text {net }}=\alpha+\delta
$$

where $\alpha$ is the vehicle angle of attack.

\section{Commanded Step Increase in Lifting Surface Deflection Angle}

For the first example case of a change in lifting surface deflection angle, the input command is taken to be a step change in deflection angle that is applied at $0.01 \mathrm{~s}$ into the simulation. The input step command corresponds to an instantaneous increase in the desired value of $\delta$ from 0 to $3.9 \mathrm{deg}$. Because the simulations are carried out for $\alpha=0.1 \mathrm{deg}$, the final value of $\delta$ is chosen to be $3.9 \mathrm{deg}$ such that the total flow angle with respect to the lifting surface remains within the bounds on $\alpha_{\text {net }}$ given in Table 2. The functional form of the input command is given by

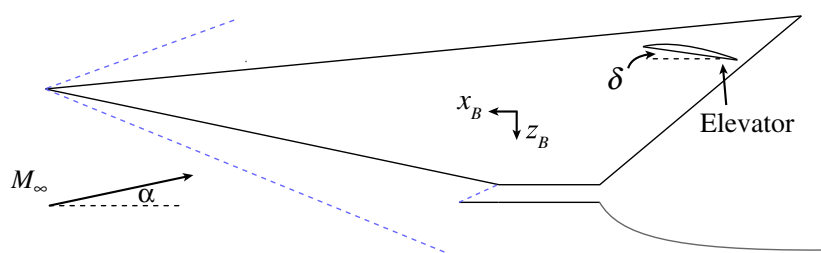

Fig. 18 Schematic illustrating geometry associated with lifting surface deflection angle and vehicle angle of attack. 


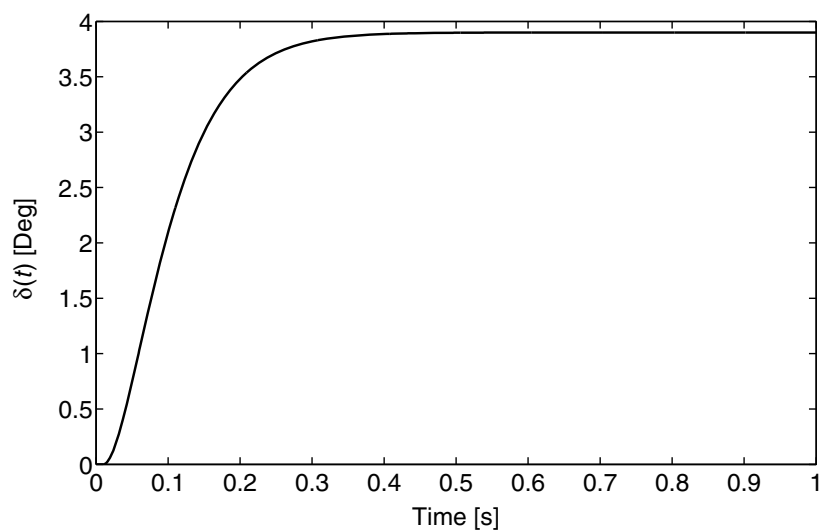

Fig. 19 Time history of applied lifting surface deflection angle $\delta(t)$.

$$
\delta_{\text {cmd }}(t)=20 H(t-\tau)\left[3.9 \operatorname{deg} \frac{\pi}{180 \mathrm{deg}}\right]
$$

where $H(t-\tau)$ is the Heaviside step function and $\tau$ is the time delay, which is taken to be $0.01 \mathrm{~s}$ in this case. A plot showing $\delta(t)$ based on the input command of Eq. (36) is given in Fig. 19, where $\delta$ has been converted from radians to degrees for visualization purposes.

Simulations are carried out using the input command described earlier at $M_{\infty}=6.5$ and $h=35 \mathrm{~km}$. As was done in the previous section, the lifting surface is first heated for $400 \mathrm{~s}$ at this Mach number and altitude at $\alpha=0.1 \mathrm{deg}$ and $\delta=0 \mathrm{deg}$. Using the temperature distribution obtained at the end of the $400 \mathrm{~s}$ period, the lifting surface is brought to aerothermoelastic equilibrium, and the simulation is then started from initial time. The vehicle angle of attack $\alpha$ is held at
$0.1 \mathrm{deg}$ throughout the simulation, and $\delta$ is given by the time history shown in Fig. 19. The entries of $x_{u}$ corresponding to the $z$ displacements of node 37 (located at the bottom surface, tip, leading edge) and node 475 (located on the bottom surface, trailing edge) are plotted as a function of time in Fig. 20. Note that $x_{u}$ includes both the constraint motion due to the change in lifting surface deflection angle $x_{u}^{C}$, as well as the elastic motion relative to the constraint motion $x_{u}^{E}$, as given in Eq. (8). Comparing the CS static and CS dynamic cases for the time range $0.5 \leq t \leq 2.0 \mathrm{~s}$, it is found that exclusion of lifting surface inertia results in a maximum absolute percent error of $5.6 \%$ for the node $37 z$ displacements and $2.3 \%$ for the node $475 z$ displacements.

To remove the effect of the constraint displacements due to the lifting surface deflection angle, the elastic displacements relative to the constraint motion $x_{u}^{E}$ are also analyzed. The entries of $x_{u}^{E}$ corresponding to the $z$ displacements of nodes 37 and 475 are plotted in Fig. 21. By isolating the elastic component of the displacements, the effect of lifting surface inertia on its response can be seen more clearly. For the CS static case, there is a small monotonic increase in elastic displacements over time due to increased steady aerodynamic loads and thermal loads as $\delta$ is increased. Examining the CS dynamic results, it is observed that the change in lifting surface deflection angle induces noticeable structural dynamic oscillations about the statically deformed state. Comparing the CS dynamic case with the CS static case for the time range $0.5 \leq t \leq 2.0 \mathrm{~s}$, inclusion of lifting surface inertia results in elastic displacements of up to 1.7 and 1.9 times the corresponding static displacements for nodes 37 and 475, respectively. The maximum absolute elastic displacements in the CS dynamic case for the complete time histories shown in Figs. 21a and $21 \mathrm{~b}$ are $0.0062 \mathrm{~m}$ (0.24 in.) for node 37 , and $0.0053 \mathrm{~m}(0.21 \mathrm{in}$. $)$ for node 475.

Although examination of the displacement time histories of the lifting surface provides insight into the response of the system, one of

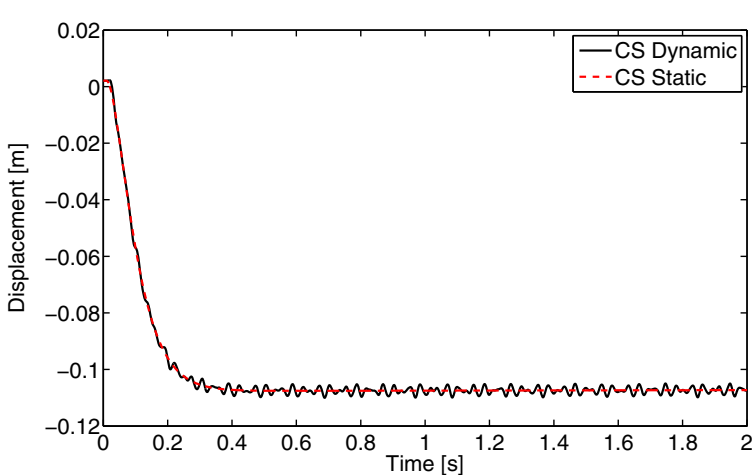

b) Node 475 (bottom surface, tip, trailing edge) a) Node 37 (bottom surface, tip, leading edge)

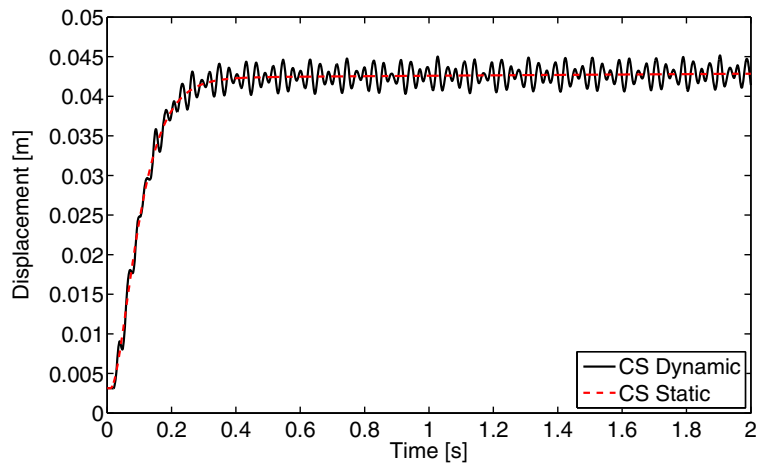

Fig. 20 Lifting surface total displacements $x_{u}$ in $z$ direction under commanded change in deflection angle with and without lifting surface inertial effects for two selected nodes.

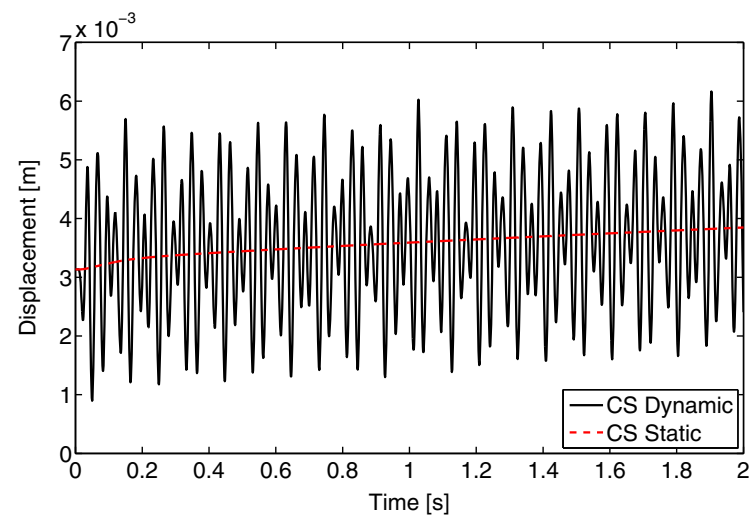

a) Node 37 (bottom surface, tip, leading edge)

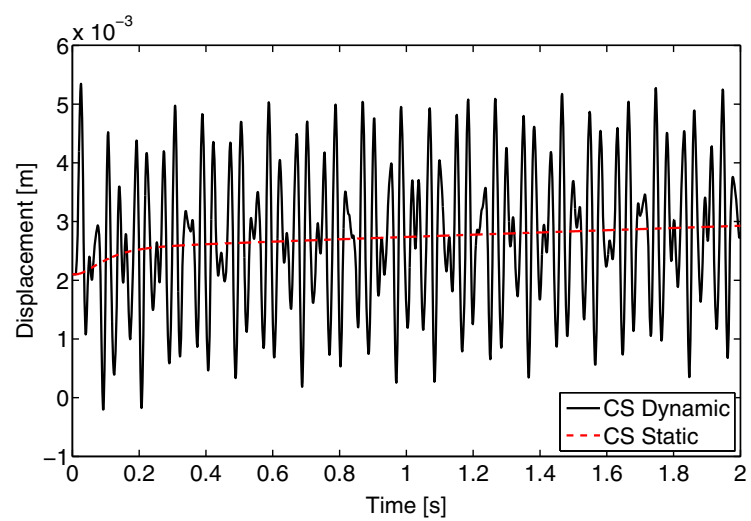

b) Node 475 (bottom surface, tip, trailing edge)

Fig. 21 Lifting surface elastic displacements $x_{u}^{E}$ in $z$ direction under commanded change in deflection angle with and without lifting surface inertial effects. 


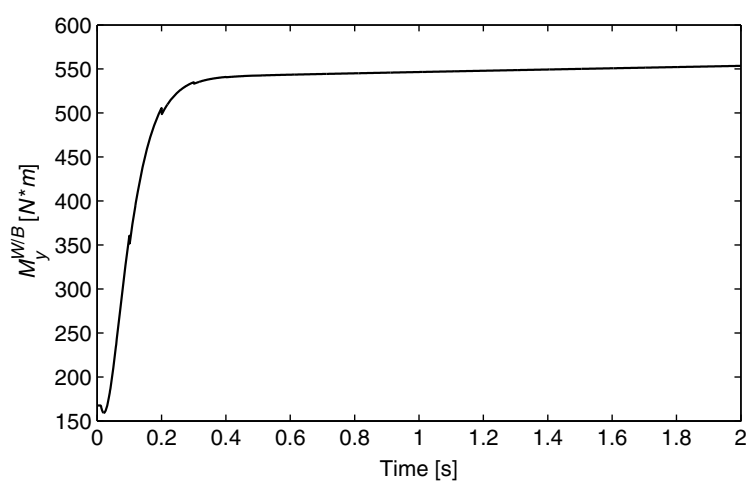

a) Static lifting surface case

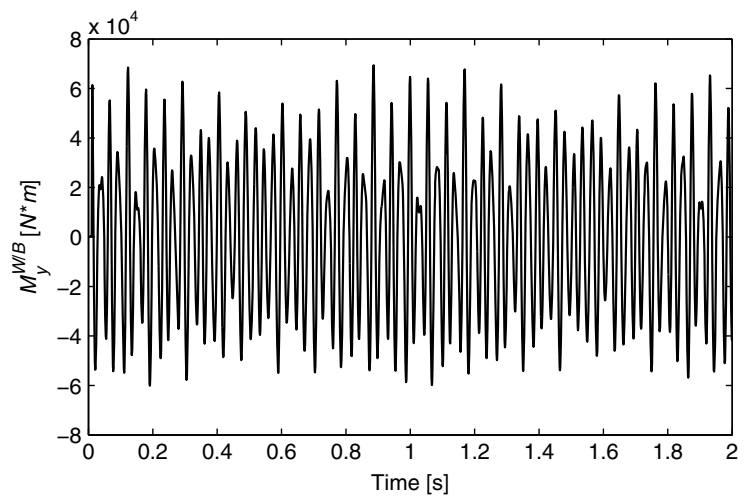

b) Dynamic lifting surface case

Fig. 22 Time-domain response of $M_{y}^{W / B}$ for both static and dynamic lifting surface cases.

the main goals of this study is to assess the impact of lifting surface inertia on the overall dynamics of the HSV. As discussed at the beginning of the current section, an important result of lifting surface-fuselage inertial coupling is that the response of the vehicle pitch rate to changes in elevator deflection angle can be adversely affected. As a step toward quantifying such effects, the pitching moments exerted by the lifting surface on the fuselage at the attachment point $M_{y}^{W / B}$ are examined for this case. The time histories of $M_{y}^{W / B}$ for the static and dynamic lifting surface cases are given in Figs. $22 \mathrm{a}$ and $22 \mathrm{~b}$, respectively. Note that a positive value of $M_{y}^{W / B}$ corresponds to a nose-up pitching moment due to the orientation of the lifting surface coordinate system. For the static lifting surface case, as $\delta$ approaches the commanded value of the deflection angle, $M_{y}^{W / B}$ asymptotically approaches a higher value. However, for the dynamic lifting surface case, the increase in $M_{y}^{W / B}$ over time is essentially indiscernible due to oscillations resulting from lifting surface inertia. For the time range $0.5 \leq t \leq 2.0 \mathrm{~s}$, the maximum absolute ratio of $M_{y}^{W / B}$ from the dynamic lifting surface case to that from the static lifting surface case is 127 , indicating that lifting surface inertia can significantly impact the pitching moment from the lifting surface under commanded changes in lifting surface deflection angle. Therefore, exclusion of lifting surface inertia may result in errors in vehicle pitch response prediction.

For the static lifting surface case, $M_{y}^{W / B}$ initially drops when the commanded change in $\delta$ is first applied at $0.01 \mathrm{~s}$. This is due to the velocity induced as the lifting surface begins to rotate about the hinge line. This velocity leads to unsteady aerodynamic pressure loads, which counteract the positive moment caused by the steady pressure loads. Once $\delta$ reaches a certain deflection angle, the negative moment caused by the unsteady aerodynamic pressure loads is balanced by the positive moment due to the steady pressure loads, and $M_{y}^{W / B}$ begins to increase. Additionally, small discontinuities in $M_{y}^{W / B}$ are

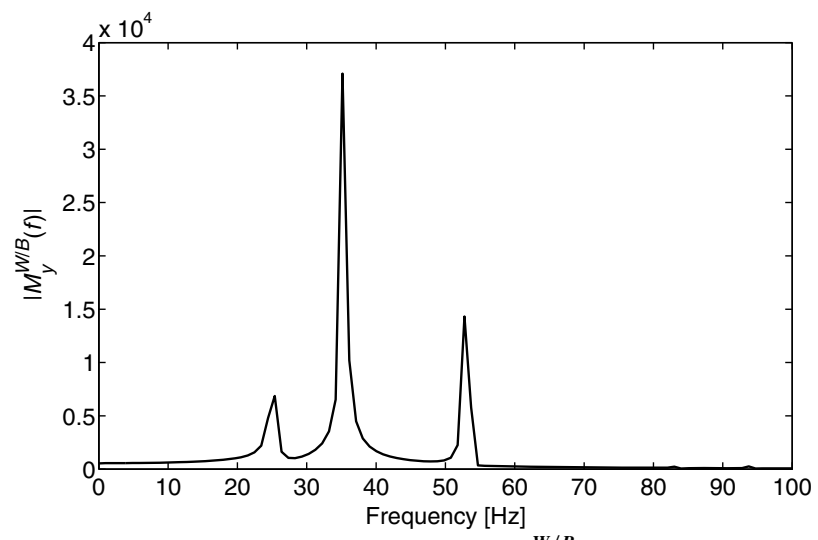

Fig. 23 Frequency-domain response of $M_{y}^{W / B}$ for dynamic lifting surface case. observed for the static lifting surface case at $0.1,0.2$, and $0.3 \mathrm{~s}$. These discontinuities are a result of the updating of the skin friction, which occurs along with the updating of the thermal boundary conditions in intervals of $0.1 \mathrm{~s}$.

To assess the frequency content of the $M_{y}^{W / B}$ time history, the FFT of the dynamic lifting surface case is taken for the time range $0.5 \leq t \leq 1.523 \mathrm{~s}$. The resulting single-sided amplitude spectrum is given in Fig. 23. As shown in the figure, there exist three main lifting surface structural dynamic modes that contribute to the $M_{y}^{W / B}$ response. The frequencies of the first three peaks are approximately 25.4, 35.2, and $52.7 \mathrm{~Hz}$. To identify which lifting surface modes correspond to these three peaks, the first four lifting surface free vibration mode shapes and frequencies are computed at the corresponding thermal state. This thermal state is obtained by heating the structure for $400 \mathrm{~s}$ at the following flight conditions: $M_{\infty}=6.5$, $\alpha=0.1 \mathrm{deg}, \delta=0 \mathrm{deg}$, and $h=35 \mathrm{~km}$. The resulting mode shapes and their frequencies are given in Fig. 24 . Based on the frequencies given in Fig. 24, the first, second, and third peaks in Fig. 23 roughly correspond with lifting surface modes one, two, and three or four, respectively. The dominant peak in Fig. 23 is the second peak, which corresponds roughly with lifting surface mode two. Examining the mode shape of lifting surface mode two, it is clear that this mode has a significant torsion component about the attachment location, and one would therefore expect that this mode would contribute most significantly to the $M_{y}^{W / B}$ dynamic response. Thus, if one were designing a control system aimed at stabilizing the HSV pitching moment, the second lifting surface mode would be the most critical mode to control.

\section{Commanded Step Increase and Decrease in Lifting Surface Deflec- tion Angle}

The next case analyzed consists of a commanded step increase in $\delta$ of $3.9 \mathrm{deg}$ issued at $0.01 \mathrm{~s}$ into the transient and a subsequent command to return $\delta$ to $0 \mathrm{deg}$ issued at $0.4 \mathrm{~s}$ into the transient. The input command $\delta_{\text {cmd }}$ for this case is given by

$$
\begin{gathered}
\delta_{\text {cmd }}(t)=20 H(t-0.01)\left[3.9 \operatorname{deg} \frac{\pi}{180 \mathrm{deg}}\right] \\
+20 H(t-0.4)\left[-3.9 \operatorname{deg} \frac{\pi}{180 \mathrm{deg}}\right]
\end{gathered}
$$

A plot showing $\delta(t)$ based on the input command of Eq. (37) is given in Fig. 25, where $\delta$ has been converted from radians to degrees for visualization purposes. As in the previous case, the flight conditions for this case are $M_{\infty}=6.5, h=35 \mathrm{~km}$, and $\alpha=0.1 \mathrm{deg}$. Again, the lifting surface is first heated at these flight conditions for $400 \mathrm{~s}$ at the initial lifting surface deflection angle $\delta=0 \mathrm{deg}$. The lifting surface is then brought to aerothermoelastic equilibrium before beginning time marching.

The entries of $x_{u}$ corresponding to the $z$-direction displacements of node 37 (located on the bottom surface, tip, leading edge) and node 


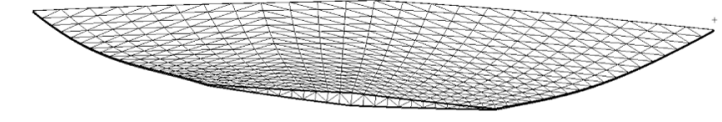

$z$

$x \quad y$

a) Mode 1: $25.1 \mathrm{~Hz}$

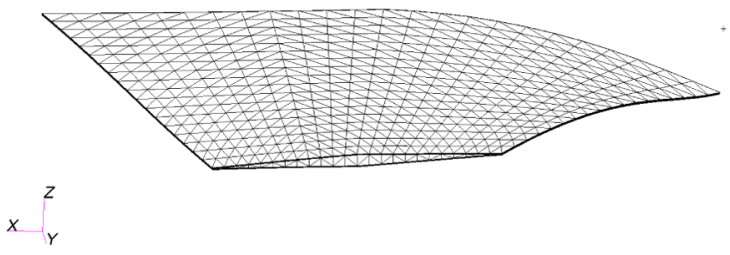

c) Mode 3: $53.2 \mathrm{~Hz}$

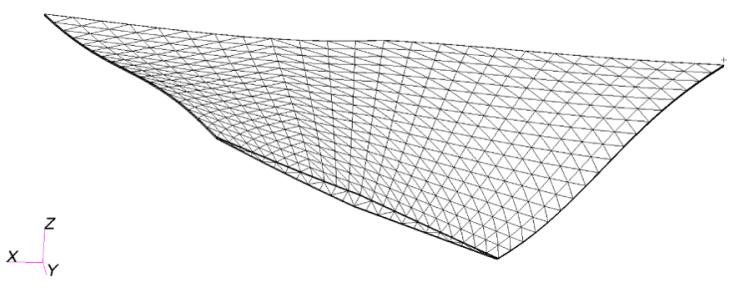

b) Mode 2: $35.6 \mathrm{~Hz}$

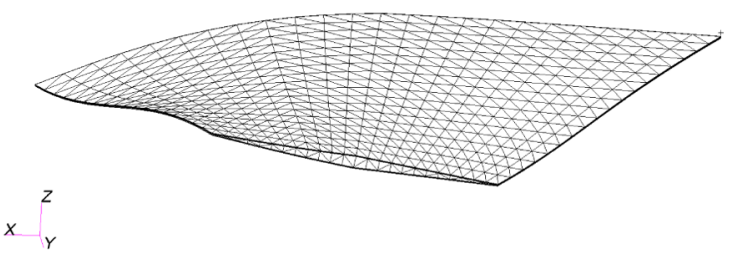

d) Mode 4: $54.3 \mathrm{~Hz}$

Fig. 24 First four free vibration modes evaluated at thermal state obtained by heating structure for $400 \mathrm{~s}$ at $M_{\infty}=6.5, \alpha=0.1 \mathrm{deg}, \delta=0 \mathrm{deg}$, and $h=35 \mathrm{~km}$.

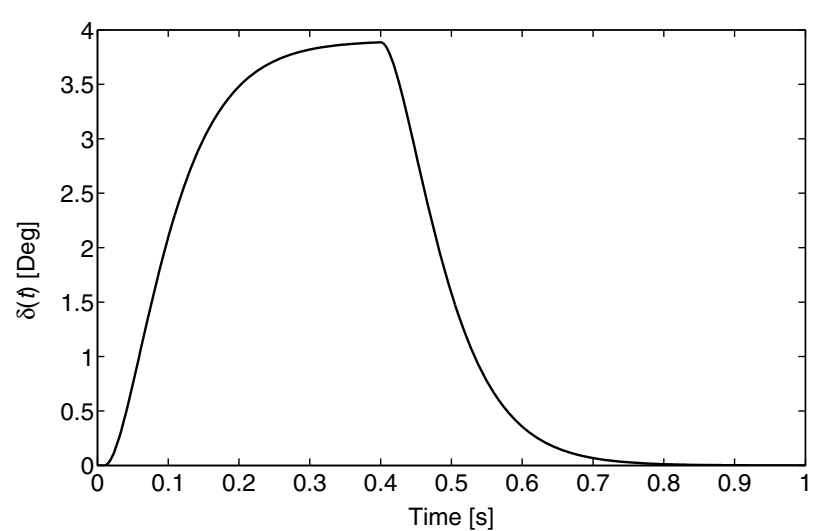

Fig. 25 Time history of applied lifting surface deflection angle $\delta(t)$.

475 (located on the bottom surface, tip, trailing edge) are given in Figs. 26a and 26b, respectively, for both the dynamic and static lifting surface cases. Recall that $x_{u}$ contains both the constraint motion due to enforced rotation of the lifting surface about the hinge line as well as elastic deformation relative to the constraint motion. To remove the displacement components due to constraint motion, the elastic displacements $x_{u}^{E}$ of nodes 37 and 475 are plotted in Figs. 27a and $27 \mathrm{~b}$, respectively, for both the dynamic and static lifting surface cases. For the CS static case in both figures, a slight increase in elastic displacement up to $0.4 \mathrm{~s}$ is observed as $\delta$ is increased and the aerodynamic pressure loads increase. As $\delta$ begins to return back to $0 \mathrm{deg}$ starting at $0.4 \mathrm{~s}$, the aerodynamic pressure loads decrease and the elastic displacements decrease slightly beginning at $0.4 \mathrm{~s}$. The CS dynamic results in both figures illustrate the effect of lifting surface structural dynamics, which result in significant oscillations in the elastic displacements about the static values. For the time range considered, inclusion of lifting surface inertia results in elastic displacements of up to 1.9 and 2.5 times the corresponding static elastic displacements for nodes 37 and 475, respectively.

Plots of the pitching moment exerted by the lifting surface on the fuselage $M_{y}^{W / B}$ are given in Figs. 28a and 28b for the static and dynamic lifting surface cases, respectively. Examining Fig. 28a, the static pitching moment results follow approximately the same trend as that of the time history of $\delta$ given in Fig. 25. However, the static value of $M_{y}^{W / B}$ initially decreases when $\delta$ first begins to increase at initial time and initially increases when $\delta$ first begins to return back to 0 deg at $0.4 \mathrm{~s}$. As discussed before, this effect is due to the unsteady aerodynamic loads induced by the instantaneous velocity of the lifting surface at these time instants. Additionally, slight discontinuities are observed in Fig. 28a in intervals of $0.1 \mathrm{~s}$, again due to the updating of the skin friction coefficients that is performed each time the thermal boundary conditions are updated. Examining Fig. 28b, significant oscillations in $M_{y}^{W / B}$ are found to occur when lifting surface inertia is included. For the time range considered, the maximum absolute ratio of $M_{y}^{W / B}$ from the dynamic lifting surface case to that from the static lifting surface case is 528 , again indicating the significant role of lifting surface inertia with regard to the pitching moment it generates on the fuselage.

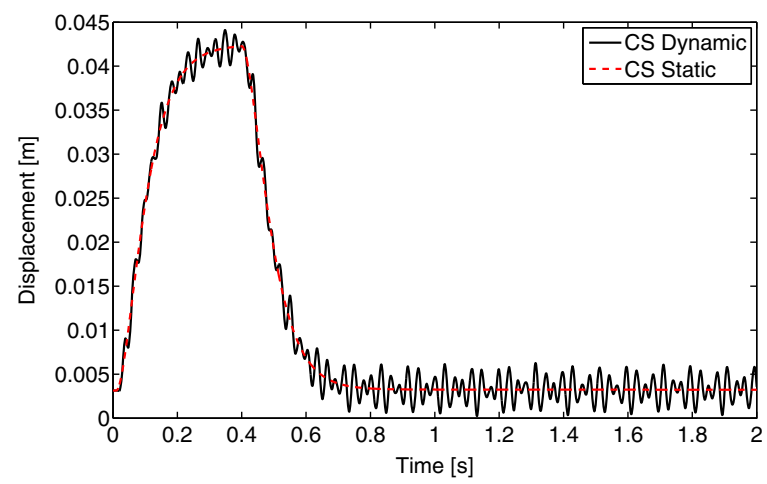

a) Node 37 (bottom surface, tip, leading edge)

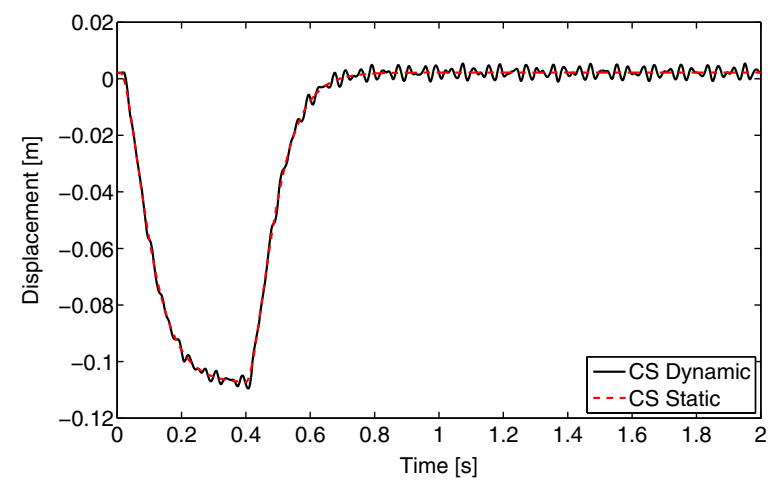

b) Node 475 (bottom surface, tip, trailing edge)

Fig. 26 Lifting surface total displacements $x_{u}$ in $z$ direction under commanded change in deflection angle with and without lifting surface inertial effects for two selected nodes. 


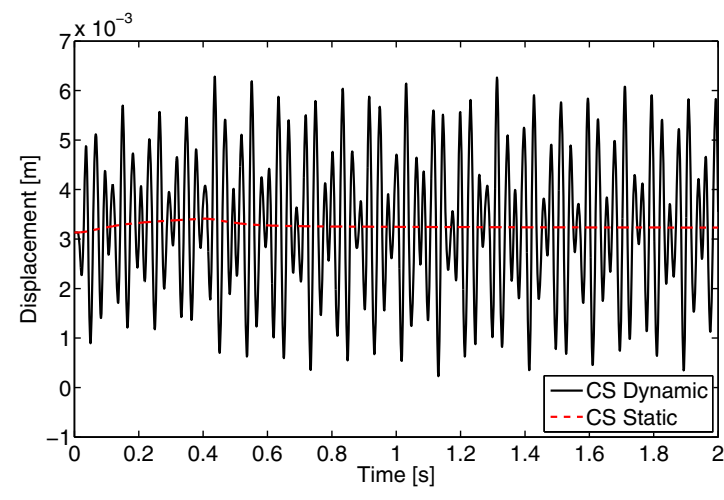

a) Node 37 (bottom surface, tip, leading edge)

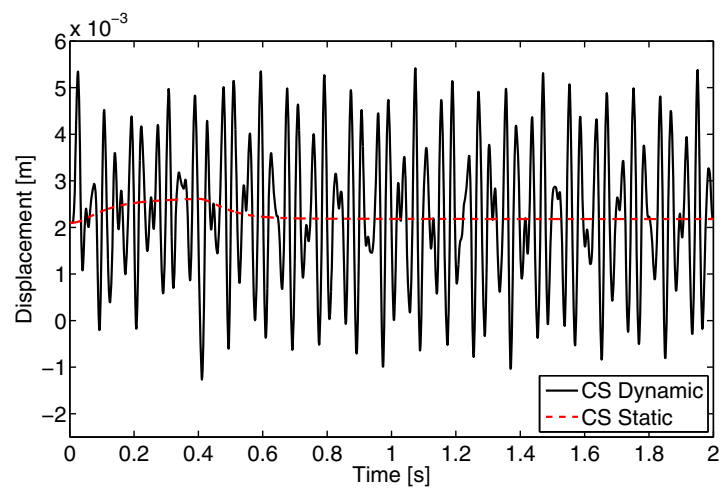

b) Node 475 (bottom surface, tip, trailing edge)

Fig. 27 Lifting surface elastic displacements $x_{u}^{E}$ in $z$ direction under commanded change in deflection angle with and without lifting surface inertial effects.

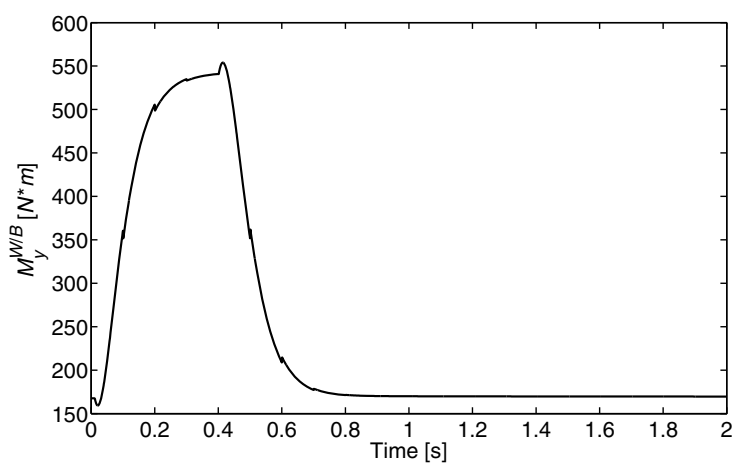

a) Static lifting surface case

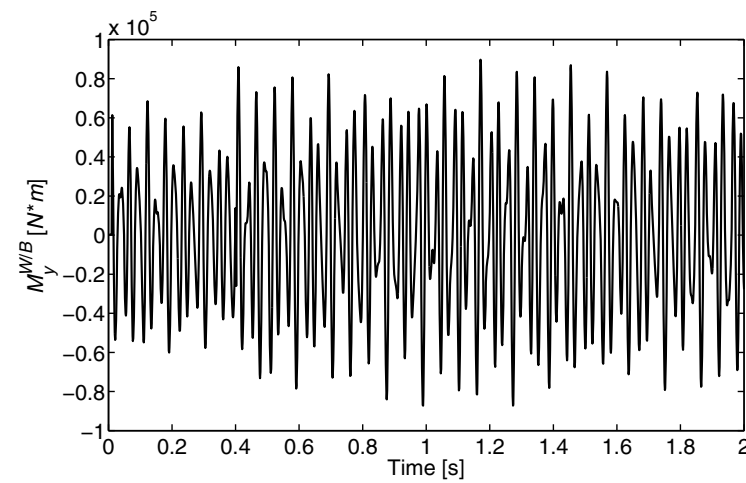

b) Dynamic lifting surface case

Fig. 28 Time-domain response of $M_{y}^{W / B}$ for both static and dynamic lifting surface cases.

\section{Conclusions}

A partitioned solution methodology was presented in which the fuselage and lifting surface are each modeled independently and information is exchanged between the two within each aeroelastic time step to bring the systems into equilibrium. This approach is advantageous in that each component can be treated as a black box with respect to the other, and thus coupling of the models is reduced to input/output exchanges of information.

Using the partitioned solution scheme, the effect of lifting surface inertial loads on overall vehicle response was examined for a configuration in which the fuselage was represented as a single-DOF oscillator having a translational displacement degree of freedom in the $z$ direction. Comparison between a simulation that included lifting surface structural dynamics and a simulation that only used a static lifting surface solution showed that exclusion of lifting surface inertia results in an $11 \%$ overprediction in the frequency of the dominant fuselage oscillation component. Additionally, the loads exerted by the lifting surface on the fuselage at the attachment point were investigated. Based on the results presented, exclusion of lifting surface inertia was found to result in an error in attachment point loads by up to a factor of 8 . However, the extent to which these attachment point loads impact fuselage response was found to be highly dependent on fuselage inertia.

To assess the impact of lifting surface inertial effects resulting from lifting surface rotation, a control input with actuator dynamics corresponding to lifting surface deflection angle was incorporated into the framework. Simulations were carried out for two different time histories of the commanded lifting surface deflection angle: 1) a commanded step increase in lifting surface deflection angle and 2) a commanded step increase followed by a subsequent step decrease in lifting surface deflection angle. The resulting pitching moments exerted by the lifting surface on the fuselage through the attachment point were examined. Results indicated that inclusion of lifting surface inertia results in departure of the instantaneous pitching moment from the lifting surface by up to a factor of 130 for the first case and 530 for the second case when compared against the static lifting surface solution.

\section{Acknowledgments}

This work was supported by the Michigan-Air Force Research Laboratory Collaborative Center in Control Science under grant FA 8650-07-2-3744 (Air Force Research Laboratory/Air Vehicles Directorate) with Michael Bolender as program manager. The authors would like to acknowledge Scott G. V. Frendreis for his contributions regarding the fuselage representation and equations of motion.

\section{References}

[1] Anderson, J. D., Jr., Hypersonic and High-Temperature Gas Dynamics, AIAA, Reston, VA, 2000, pp. 9-11.

[2] Bolender, M. A., and Doman, D. B., "Nonlinear Longitudinal Dynamical Model of an Air-Breathing Hypersonic Vehicle," Journal of Spacecraft and Rockets, Vol. 44, No. 2, March-April 2007, pp. 374387.

doi: $10.2514 / 1.23370$

[3] Chavez, F. R., and Schmidt, D. K., "Analytical Aeropropulsive/ Aeroelastic Hypersonic-Vehicle Model with Dynamic Analysis," Journal of Guidance, Control, and Dynamics, Vol. 17, No. 6, MarchApril 2007, pp. 1308-1319. doi: $10.2514 / 3.21349$

[4] Bertin, J. J., Hypersonic Aerothermodynamics, AIAA, Reston, VA, 1994, pp. 1-3.

[5] Schmidt, D. K., "Dynamics and Control of Hypersonic Aeropropulsive/ Aeroelastic Vehicles," Proceedings of the 1992 AIAA Guidance, Navigation, and Control Conference, AIAA Paper 1992-4326, 1992.

[6] McRuer, D., "Design and Modeling Issues for Integrated Airframe/ Propulsion Control of Hypersonic Flight Vehicles," Proceedings of the 
1991 American Control Conference, American Automatic Control Council, Evanston, IL, June 1991, pp. 729-734.

[7] Bilimoria, K. D., and Schmidt, D. K., "Integrated Development of the Equations of Motion for Elastic Hypersonic Vehicles," Journal of Guidance, Control, and Dynamics, Vol. 18, No. 1, Jan.-Feb. 1995, pp. 73-81. doi: $10.2514 / 3.56659$

[8] Parker, J. T., Serrani, A., Yurkovich, S., Bolender, M. A., and Doman, D. B., "Control-Oriented Modeling of an Air-Breathing Hypersonic Vehicle," Journal of Guidance, Control, and Dynamics, Vol. 30, No. 3, May-June 2007, pp. 856-869. doi: $10.2514 / 1.27830$

[9] Bolender, M. A., "An Overview on Dynamics and Controls Modelling of Hypersonic Vehicles," Proceedings of the 2009 American Control Conference, IEEE Publ., Piscataway, NJ, June 2009, pp. 2507-2512.

[10] Frendreis, S. G. V., and Cesnik, C. E. S., "3D Simulation of a Flexible Hypersonic Vehicle," Proceedings of the 2010 AIAA Atmospheric Flight Mechanics Conference, AIAA Paper 2010-8229, 2010.

[11] Falkiewicz, N. J., and Cesnik, C. E. S., "A Reduced-Order Modeling Framework for Integrated Thermo-Elastic Analysis of Hypersonic Vehicles," Proceedings of the 50th AIAA/ASME/ASCE/AHS/ASC Structures, Structural Dynamics, and Materials Conference, AIAA Paper 2009-2308, 2009.

[12] Falkiewicz, N. J., and Cesnik, C. E. S., "Proper Orthogonal Decomposition for Reduced-Order Thermal Solution in Hypersonic Aerothermoelastic Simulations," AIAA Journal, Vol. 49, No. 5, May 2011, pp. 994-1009. doi:10.2514/1.J050701

[13] Falkiewicz, N. J., Cesnik, C. E. S., Bolender, M. A., and Doman, D. B., "Thermoelastic Formulation of a Hypersonic Vehicle Control Surface for Control-Oriented Simulation," Proceedings of the 2009 AIAA Guidance, Navigation, and Control Conference, AIAA Paper 2009$6284,2009$.

[14] Falkiewicz, N. J., Cesnik, C. E. S., Crowell, A. R., and McNamara, J. J., "Reduced-Order Aerothermoelastic Framework for Hypersonic Vehicle Control Simulation," AIAA Journal, Vol. 49, No. 8, Aug. 2011, pp. $1625-1646$. doi:10.2514/1.J050802

[15] Falkiewicz, N. J., and Cesnik, C. E. S., "Enhanced Modal Solutions for Structural Dynamics in Aerothermoelastic Analysis," Proceedings of the 52nd AIAA/ASME/ASCE/AHS/ASC Structures, Structural Dynamics, and Materials Conference, AIAA Paper 2011-1963, 2011.

[16] Eckert, E. R. G., "Engineering Relations for Friction and Heat Transfer to Surfaces in High Velocity Flow," Journal of the Aeronautical Sciences, Vol. 22, No. 8, March 1955, pp. 585-587.

[17] Bolender, M. A., and Doman, D. B., "Nonlinear Longitudinal Dynamical Model of an Air-Breathing Hypersonic Vehicle," Journal of Spacecraft and Rockets, Vol. 44, No. 2, 2007, pp. 374-387. doi: $10.2514 / 1.23370$
[18] Dalle, D. J., Frendreis, S. G. V., Driscoll, J. F., and Cesnik, C. E. S., "Hypersonic Vehicle Flight Dynamics with Coupled Aerodynamics and Reduced-Order Propulsive Models," Proceedings of the 2010 AIAA Atmospheric Flight Mechanics Conference, AIAA Paper 2010-7930, 2010.

[19] Falkiewicz, N. J., and Cesnik, C. E. S., "Proper Orthogonal Decomposition for Reduced-Order Thermal Solution in Hypersonic Aerothermoelastic Simulations," Proceedings of the 51 st AIAA/ASME/ ASCE/AHS/ASC Structures, Structural Dynamics, and Materials Conference, AIAA Paper 2010-2798, 2010.

[20] Wilson, E. L., Yuan, M.-W., and Dickens, J. M., "Dynamic Analysis by Direct Superposition of Ritz Vectors," Earthquake Engineering \& Structural Dynamics, Vol. 10, No. 6, Nov.-Dec. 1982, pp. 813-821. doi:10.1002/eqe.4290100606

[21] Craig, R. R., and Kurdila, A. J., Fundamentals of Structural Dynamics, 2nd ed., Wiley, Hoboken, NJ, 2006, p. 228

[22] "MSC Nastran Basic Dynamic Analysis User's Guide," Ver. 68, MSC Software Corp., Santa Ana, CA, 2004, p. 151.

[23] Heath, M. T., Scientific Computing: An Introductory Survey, 2nd ed., McGraw-Hill, New York, 2002, pp. 223, 501.

[24] Vogel, J. M., Kelkar, A. G., Inger, G., Whitmer, C., Sidlinger, A., and Rodriguez, A., "Control-Relevant Modeling of Hypersonic Vehicles," Proceedings of the 2009 American Control Conference, IEEE Publ., Piscataway, NJ, June 2009, pp. 2519-2524.

[25] Pendleton, E., Moster, G., and Keller, D., "Transonic Aeroelastic Models of Highly Swept Hypersonic Lifting Surfaces," Journal of Aircraft, Vol. 32, No. 6, Nov.-Dec. 1995, pp. 1169-1176. doi: $10.2514 / 3.46860$

[26] Oppenheimer, M. W., and Doman, D. B., "A Hypersonic Vehicle Model Developed with Piston Theory," Proceedings of the 2006 AIAA Atmospheric Flight Mechanics Conference, AIAA Paper 2006-6637, 2006.

[27] McNamara, J. J., Friedmann, P. P., Powell, K. G., Thuruthimattam, B. J., and Bartels, R. E., "Aeroelastic and Aerothermoelastic Behavior in Hypersonic Flow," AIAA Journal, Vol. 46, No. 10, Oct. 2008, pp. 25912610. doi: $10.2514 / 1.36711$

[28] Falkiewicz, N. J., Frendreis, S. G. V., and Cesnik, C. E. S., "Effect of Control Surface-Fuselage Inertial Coupling on Hypersonic Vehicle Flight Dynamics," Proceedings of the 2011 AIAA Atmospheric Flight Mechanics Conference, AIAA Paper 2011-6378, 2011.

[29] Gibson, T. E., Crespo, L. G., and Annaswamy, A. M., "Adaptive Control of Hypersonic Vehicles in the Presence of Modeling Uncertainties," Proceedings of the 2009 American Control Conference, IEEE Publ., Piscataway, NJ, June 2009, pp. 3178-3183.

\author{
R. Ganguli \\ Associate Editor
}

